\title{
A whole-brain monosynaptic input connectome to neuron classes in mouse visual cortex
}

\section{Shenqin Yao}

Allen Institute for Brain Science

\section{Quanxin Wang}

The Allen Institute for Brain Science

Karla Hirokawa

Allen Institute for Brain Science

\section{Benjamin Ouellette}

Allen Institute for Brain Science

\section{Ruweida Ahmed}

Allen Institute for Brain Science Jasmin Bomben

Allen Institute for Brain Science Krissy Brouner

Allen Institute for Brain Science Linzy Casal

Allen Institute for Brain Science

\section{Shiella Caldejon}

Allen Institute for Brain Science

Andy Cho

Allen Institute for Brain Science

\section{Nadezhda Dotson}

Allen Institute for Brain Science

\section{Tanya Daigle}

Allen Institute for Brain Science

\section{Tom Egdorf}

Allen Institute for Brain Science

\section{Rachel Enstrom}

Allen Institute for Brain Science

\section{Amanda Gary}

Allen Institute for Brain Science

Emily Gelfand

Allen Institute for Brain Science 
Allen Institute for Brain Science

\section{Fiona Griffin}

Allen Institute for Brain Science

\section{Hong Gu}

Allen Institute for Brain Science

Nicole Hancock

Allen Institute for Brain Science

\section{Robert Howard}

Allen Institute for Brain Science

\section{Leonard Kuan}

Allen Institute for Brain Science

\section{Sophie Lambert}

Allen Institute for Brain Science

\section{Eric Lee}

Allen Institute for Brain Science Jennifer Luviano

Allen Institute for Brain Science

\section{Kyla Mace}

Allen Institute for Brain Science

\section{Michelle Maxwell}

Allen Institute for Brain Science

\section{Marty Mortrud}

Allen Institute for Brain Science

\section{Maitham Naeemi}

Allen Institute for Brain Science

Chelsea Nayan

Nhan-Kiet Ngo

Allen Institute for Brain Science

\section{Thuyanh Nguyen}

Allen Institute for Brain Science

\section{Kat North}

Allen Institute for Brain Science

\section{Shea Ransford}

Allen Institute for Brain Science

\section{Augustin Ruiz}

Allen Institute https://orcid.org/0000-0002-8361-5152

\section{Sam Seid}

Allen Institute for Brain Science 
Allen Institute for Brain Science

\section{Michael Taormina}

Allen Institute for Brain Science

\section{Wayne Wakeman}

Allen Institute for Brain Science

Thomas Zhou

Allen Institute for Brain Science

Philip Nicovich

Allen Institute for Brain Science

\section{Ali Williford}

Allen Institute for Brain Science

\section{Lydia Potekhina}

Allen Institute for Brain Science

\section{Medea McGraw}

Allen Institute for Brain Science

\section{Lydia $\mathrm{Ng}$}

Allen Institute for Brain Science

\section{Peter Groblewski}

Allen Institute for Brain Science

\section{Bosiljka Tasic}

Allen Institute for Brain Science https://orcid.org/0000-0002-6861-4506

\section{Stefan Mihalas}

Allen Institute for Brain Science https://orcid.org/0000-0002-2629-7100

Julie Harris

Allen Institute for Brain Science

\section{Ali Cetin}

Allen Institute for Brain Science

Hongkui Zeng ( $\square$ hongkuiz@alleninstitute.org )

Allen Institute for Brain Science https://orcid.org/0000-0002-0326-5878

\section{Resource}

Keywords: neurons, brain function, brain-wide inputs, rabies virus tracing

Posted Date: October 15th, 2021

DOI: https://doi.org/10.21203/rs.3.rs-953043/v1

License: (c) (i) This work is licensed under a Creative Commons Attribution 4.0 International License. Read Full License 



\section{A whole-brain monosynaptic input connectome to neuron classes in mouse visual cortex}

3 Shenqin Yao $^{1 *}$, Quanxin Wang ${ }^{1}$, Karla E. Hirokawa ${ }^{1 \dagger}$, Benjamin Ouellette ${ }^{1}$, Ruweida Ahmed ${ }^{1}$, 4 Jasmin Bomben ${ }^{1}$, Krissy Brouner ${ }^{1}$, Linzy Casal ${ }^{1}$, Shiella Caldejon ${ }^{1}$, Andy Cho ${ }^{1}$, Nadezhda I. 5 Dotson $^{1}$, Tanya L. Daigle ${ }^{1}$, Tom Egdorf ${ }^{1}$, Rachel Enstrom ${ }^{1}$, Amanda Gary ${ }^{1}$, Emily Gelfand ${ }^{1}$, 6 Melissa Gorham ${ }^{1}$, Fiona Griffin ${ }^{1}$, Hong Gu${ }^{1}$, Nicole Hancock ${ }^{1}$, Robert Howard ${ }^{1}$, Leonard Kuan ${ }^{1}$, 7 Sophie Lambert ${ }^{1}$, Eric Kenji Lee ${ }^{1}$, Jennifer Luviano ${ }^{1}$, Kyla Mace ${ }^{1}$, Michelle Maxwell ${ }^{1}$, Marty T. 8 Mortrud $^{1}$, Maitham Naeemi ${ }^{1}$, Chelsea Nayan ${ }^{1}$, Nhan-Kiet Ngo ${ }^{1}$, Thuyanh Nguyen ${ }^{1}$, Kat North ${ }^{1 \dagger}$, 9 Shea Ransford ${ }^{1}$, Augustin Ruiz ${ }^{1}$, Sam Seid ${ }^{1}$, Jackie Swapp ${ }^{1}$, Michael J Taormina ${ }^{1}$, Wayne 10 Wakeman $^{1}$, Thomas Zhou ${ }^{1}$, Philip R. Nicovich ${ }^{1 \dagger}$, Ali Williford ${ }^{1}$, Lydia Potekhina ${ }^{1}$, Medea 11 McGraw $^{1}$, Lydia Ng${ }^{1}$, Peter A. Groblewski ${ }^{1}$, Bosiljka Tasic ${ }^{1}$, Stefan Mihalas ${ }^{1}$, Julie A Harris ${ }^{1 \dagger}$, 12 Ali Cetin $^{1 \#}$, Hongkui Zeng ${ }^{{ }^{*}}$

$14{ }^{1}$ Allen Institute for Brain Science, Seattle, WA 98109, USA

15 \# Current address: CNC Program, Stanford University, Palo Alto, CA, USA

$16 \dagger$ Current address: Cajal Neuroscience, Seattle, WA 98102, USA.

17 *Correspondence: shenqiny@alleninstitute.org, and hongkuiz@alleninstitute.org 


\section{Abstract}

Identification of the structural connections between neurons is a prerequisite to 24 understanding brain function. We developed a pipeline to systematically map brain-wide 25 monosynaptic inputs to specific neuronal populations using Cre-driver mouse lines and the 26 recombinant rabies tracing system. We first improved the rabies virus tracing strategy to accurately 27 identify starter cells and to efficiently quantify presynaptic inputs. We then mapped brain-wide 28 presynaptic inputs to different excitatory and inhibitory neuron subclasses in the primary visual 29 cortex and seven higher visual areas. Our results reveal quantitative target-, layer- and cell-class30 specific differences in the retrograde connectomes, despite similar global input patterns to different 31 neuronal populations in the same anatomical area. The retrograde connectivity we define is 32 consistent with the presence of the ventral and dorsal visual information processing streams and 33 reveals further subnetworks within the dorsal stream. The hierarchical organization of the entire 34 visual cortex can be derived from intracortical feedforward and feedback pathways mediated by 35 upper- and lower-layer input neurons, respectively. This study expands our knowledge of the 36 brain-wide inputs regulating visual areas and demonstrates that our improved rabies virus tracing 37 strategy can be used to scale up the effort in dissecting connectivity of genetically defined cell 38 populations in the whole mouse brain. 


\section{Introduction}

The identity and function of neurons are determined not only by the inherent molecular

43 and physiological characteristics of individual cells, but also by the synaptic connectivity through

44 which diverse neuronal types form circuits. Advances in electron microscopy (EM) have enabled

45 the reconstruction of synaptic resolution connectomes with different complexity, from the brain of

46 C. elegans with 302 neurons to that of adult Drosophila melanogaster and larval zebrafish with

$47 \sim 100,000$ neurons $^{1-7}$. Although brain-wide connectomics at single-cell resolution is currently

48 beyond our grasp for complex nervous systems with over millions of neurons, different strategies

49 have been applied to reveal the connectivity at distinct levels of resolution. Large-scale EM has

50 been applied to reconstruct sub-volumes of the mouse and human brains, revealing both cellular

51 and sub-cellular structures ${ }^{8-12}$. Whole-brain imaging of genetically labeled neurons can reveal the

52 morphology of entire neurons and the fine details of dendritic and axonal coverage ${ }^{13,14}$.

53 Electrophysiological strategies such as multiple-patch clamp recordings reveal neurons that are

54 synaptically connected and functionally depend on each other ${ }^{15,16}$. Optogenetic activation of axon

55 terminals of presynaptic neurons coupled with whole-cell recording of postsynaptic neurons have

56 been utilized to examine neural connectivity over a range of spatial scales ${ }^{16-20}$. Wide-field imaging

57 of genetically encoded calcium indicators allows simultaneous activity-monitoring of hundreds of neurons, which do not have to be synaptically connected ${ }^{21}$. Nonetheless, due to current technical limitations, these strategies cannot be used to reveal whole-brain connectivity in complex nervous systems.

Systematic mapping of afferent connectivity to specific cell populations has been greatly aided by the introduction of the monosynaptic, retrograde trans-synaptic rabies virus system ${ }^{22,23}$.

63 Rabies glycoprotein (RG)-deleted rabies viruses can be coupled with various genetic and viral 64 tools to ensure the cell-type specific labeling of direct presynaptic inputs ${ }^{24-36}$. Many efforts have 65 been made to improve the efficiency and specificity of rabies virus tracer while reducing its toxicity, including the construction of recombinant rabies viruses from the CVS N2c virus strain ${ }^{37}$, 67 utilization of an engineered $\mathrm{RG}^{25}$, and generation of a double-deletion-mutant rabies virus ${ }^{38}$ and a self-inactivating rabies virus ${ }^{39}$. In addition, an intersectional rabies tracing strategy targeting Flpand Cre-double labeled neurons has been generated to conduct cell-type-specific circuit tracing at an even more precise level ${ }^{40}$. 
The ever-expanding repertoire of genetic and viral tools has enabled the construction of

72 brain-wide mesoscale connectomes in a reasonable time frame ${ }^{41-48}$. In our effort to build the Allen

73 Mouse Brain Connectivity Atlas, we combined viral tools, transgenic mouse lines, high-

74 throughput imaging, and informatics to map brain-wide efferent connections at the level of cell

75 classes $^{49,50}$. By delivering recombinant adeno-associated viruses (AAV) with Cre-dependent

76 expression of enhanced green fluorescent protein (EGFP) to target brain areas of Cre transgenic

77 lines, we labeled axons from selective $\mathrm{Cre}^{+}$neuronal classes and subclasses. Our informatics

78 pipeline, which includes registration of image series to the Allen Mouse Brain Common

79 Coordinate Framework (CCF) and automatic segmentation of fluorescent axonal projections ${ }^{51,52}$,

80 enabled the quantification and comparison of whole-brain projections across multiple regions and

81 cell classes. The resulting high-resolution mesoscale projection maps provide the foundation for

82 in-depth dissection of the logic of mouse brain connectivity.

83 Aiming to construct a complementary afferent map of mouse brain-wide connectivity, we

84 now developed an improved version of the monosynaptic rabies virus tracing system and

85 incorporated rabies-mediated presynaptic input mapping into our pipeline. Our system consists of

86 a single AAV helper virus that allows the accurate identification of starter neurons and rabies

87 viruses expressing nucleus-localized EGFP marker to facilitate automatic quantification of

88 presynaptic inputs. In this study, we utilized the retrograde connectome pipeline to map a brain-

89 wide, cell-class-specific, presynaptic connectome for the mouse visual cortex, including both

90 primary and higher visual areas. Mouse visual cortex contains at least ten visuotopically organized

91 cortical areas ${ }^{53-56}$. These visual areas are strongly interconnected to form a hierarchical network

92 with two visual streams as revealed by anterograde tracing ${ }^{49,57}$, similar to what have been known

93 in the primates and cat ${ }^{58}$. In primates, visual cortical hierarchy were defined by feedforward and

94 feedback connections via laminar distribution of the retrogradely labeled neurons ${ }^{59}$. It remains

95 largely unknown whether visual hierarchy and streams in mouse can also be defined with 96 retrograde tracing.

97 By applying the monosynaptic rabies tracing system to Cre driver mouse lines labeling 98 different excitatory and inhibitory neuron subclasses ${ }^{44,49}$, our results reveal quantitative target-, 99 layer- and cell-class-specific differences in the retrograde connectomes, despite similar global 100 input patterns. We find that the retrograde connectomes of the same cell classes in different target 101 areas are more different from each other than the retrograde connectomes of different cell classes 
102 in the same target area. Layer (L)-specific features are also identified, for example, L4 neurons 103 receive more thalamic inputs and fewer inputs from higher-order association cortical areas, 104 whereas L6 neurons are the main targets of contralateral/callosal inputs. Our study confirms 105 previous findings of the dorsal and ventral streams in the mouse visual cortex ${ }^{57}$ and further reveals 106 distinct subnetworks in the medial and lateral parts of the dorsal stream. Finally, our previous study 107 showed that the hierarchical organization among different areas of the mouse visual cortex can be 108 derived from axon termination patterns in the anterograde connectomes ${ }^{49}$, here we demonstrate 109 that it can also be derived from the retrograde connectomes independently, via the feedforward and feedback projections mediated by upper- and lower-layer input neurons, respectively.

\section{Results}

\section{A pipeline for the mesoscale retrograde connectome}

To systematically map the whole-brain presynaptic inputs to different cell classes, we

115 established a standardized high-throughput pipeline based on our pipeline for projection mapping 116 across the entire brain ${ }^{49,50}$, including the following steps: virus production and specimen generation

117 (Figure 1a-b), data acquisition and processing (Figure 1c-e), and post-informatics 118 characterization (Figure $1 \mathbf{f}-\mathbf{g}$ ). The monosynaptic cell-type-specific rabies tracing system consists 119 of EnvA-pseudotyped and glycoprotein-deleted rabies virus (EnvA RV ${ }^{\mathrm{dG}}$ ) expressing histone120 tagged EGFP (H2B-EGFP) and AAV helper virus conditionally expressing dTomato, the EnvA 121 receptor TVA and RG. This system was coupled with Cre-driver mouse lines to reveal inputs to 122 defined cell classes or types. The AAV helper virus and the monosynaptic rabies tracer were 123 sequentially injected to the same target site with a three-week interval, followed by imaging of the 124 whole brain one week after rabies infection. In this study focused on the visual cortex, our target 125 site identification was guided by intrinsic signal imaging (ISI) of the visual areas. Rabies-labeled 126 brains were imaged using high-throughput serial 2-photon tomography (STPT) at every $100 \mu \mathrm{m}$, 127 with a total of 140 images for each brain. Injection polygons were drawn based on the expression 128 of dTomato from the AAV helper virus, and the centroids of the injection site polygons were later 129 used to verify and assign the target site. Image series were processed in the informatics pipeline 130 for automatic segmentation of signal and registration to the Allen Mouse Brain Common 131 Coordinate Framework version $3(\mathrm{CCFv} 3)^{51}$ for subsequent data analyses. Brain sections were 132 collected after STPT and those around the injection sites were further immunostained to enhance 
133 the dTomato signal. Starter cells were quantified after confocal imaging of the stained sections.

134 Rigorous manual quality control steps were conducted to exclude experiments with noticeable tissue damage, injection failure, imaging failure or segmentation errors.

136 We re-engineered several features of pre-existing rabies tracing tools to facilitate accurate identification of starter cells and automatic quantification of presynaptic inputs ${ }^{31,37,60}$ (Figure 1a).

138 Our AAV helper virus uses the FLEX strategy to conditionally express a tricistronic cassette of 139 TVA $^{66 \mathrm{~T}}$-P2A-dTomato-P2A-RG under the control of human synapsin promoter (hSyn), and the 140 ATG of the tricistronic cassette was placed 5' to the FLEX sites $^{60}$ (Figure 1a). The co-expression 141 of TVA, dTomato, and RG from the same expression cassette allows unambiguous identification 142 of starter neurons. Both in vivo (Supplementary Figure 1a-c and Supplementary Table 1) and 143 in vitro (not shown) tests of the new AAV helper virus demonstrate that this single AAV helper, 144 with the use of the attenuated form of TVA, TVA ${ }^{66 \mathrm{~T}}$, significantly reduces spurious virus labeling 145 in the absence of Cre. Our rabies tracer is based on the CVS N2 $\mathrm{c}^{\mathrm{dG}}$ rabies strain ${ }^{37}$ and expresses 146 H2B-EGFP. Compared with the EGFP-expressing and H2B-EGFP-experssing SAD B19dG rabies viruses (Supplementary Figure 1d-f and Supplementary Table 1), the H2B-EGFP-expressing 148 CVS N2 $\mathrm{c}^{\mathrm{dG}}$ viruses mediates stringent nucleus labeling, which facilitates automatic quantification 149 of presynaptic cells by minimizing neurite labeling. This is the virus that we use throughout the 150 paper and will refer to it simply as RV-H2B-EGFP.

151 We performed several control experiments to verify the specificities of our rabies virus 152 tracing system in the absence of Cre, in the absence of RG provided by AAV helper viruses or in 153 transgenic lines in which Cre is expressed in non-neuronal cell types. We found that applying the 154 monosynaptic rabies tracing system to wild-type mice (i.e., in the absence of Cre) led to only a 155 few H2B-EGFP-labeled cells in the injection site, but no starter cells in the injection site and no 156 H2B-GFP-labeled cells outside the injection site (Supplementary Figure 2a-b, and 157 Supplementary Table 1). This shows that our system does not have the issue of spurious local 158 rabies virus uptake due to low-level expression from the AAV helper in the absence of Cre ${ }^{29,32,34,61}$, 159 or local infection by small quantities of $\mathrm{RG}$-coated $\mathrm{RV}^{\mathrm{dG}}$ virus particles that may be present in the 160 EnvA-pseudotyped rabies virus preparation.

161 We then confirmed that the trans-synaptic transfer of the recombinant rabies relies on the 162 expression of rabies $\mathrm{G}$ from the AAV helper. A G-minus version of the AAV helper virus, which 163 conditionally expresses $\mathrm{TVA}^{66 \mathrm{~T}}$ and dTomato after Cre-mediated recombination, was injected into 
$164 \mathrm{Cre}^{+}$mice, followed by the injection of rabies virus three weeks later. We observed H2B-EGFP165 labeled cells only at the injection site and nowhere else in the brain ${ }^{27}$. This finding confirms that

166 the presynaptic labeling is specific for the $\mathrm{Cre}^{+}$starter cells expressing the tricistronic cassette and 167 infected with RV-H2B-GFP rabies viruses.

168 Finally, we investigated whether rabies infection of non-neuronal cell types affects the 169 specificity of monosynaptic tracing (Supplementary Figure 2c-e and Supplementary Table 1). 170 We tested the monosynaptic rabies tracing system in three non-neuronal Cre lines, Olig2-Cre ${ }^{62}$, 171 Tek-Cre ${ }^{63}$, and Aldh111-CreERT2 ${ }^{64}$, which express Cre in oligodendrocytes, vascular endothelium, 172 and astrocytes, respectively. Among all experiments using the non-neuronal Cre lines, with either 173 the hSyn-driven AAV helper virus used in the pipeline or a similarly constructed CMV-driven 174 helper virus, sporadic long-distance H2B-EGFP-labeled cells were found only in 50\% of the 175 injected Aldh111-CreERT2 mice (Supplementary Figure 2e). Our results show that the 176 occasionally infected non-neuronal cells do not support the spread of rabies virus to neurons in 177 local or distant areas.

178 Through rigorous testing, we show that our rabies tracing strategy presents minimal non179 specific labeling in the absence of Cre and enables unambiguous identification of starter cells and 180 automatic quantification of presynaptic inputs. By combining Cre-driver lines and the improved 181 rabies tracing system, we aim to utilize our standardized pipeline for monosynaptic retrograde 182 mapping to generate a comprehensive and quantitative mesoscale input network registered into a 183 common 3D space. 

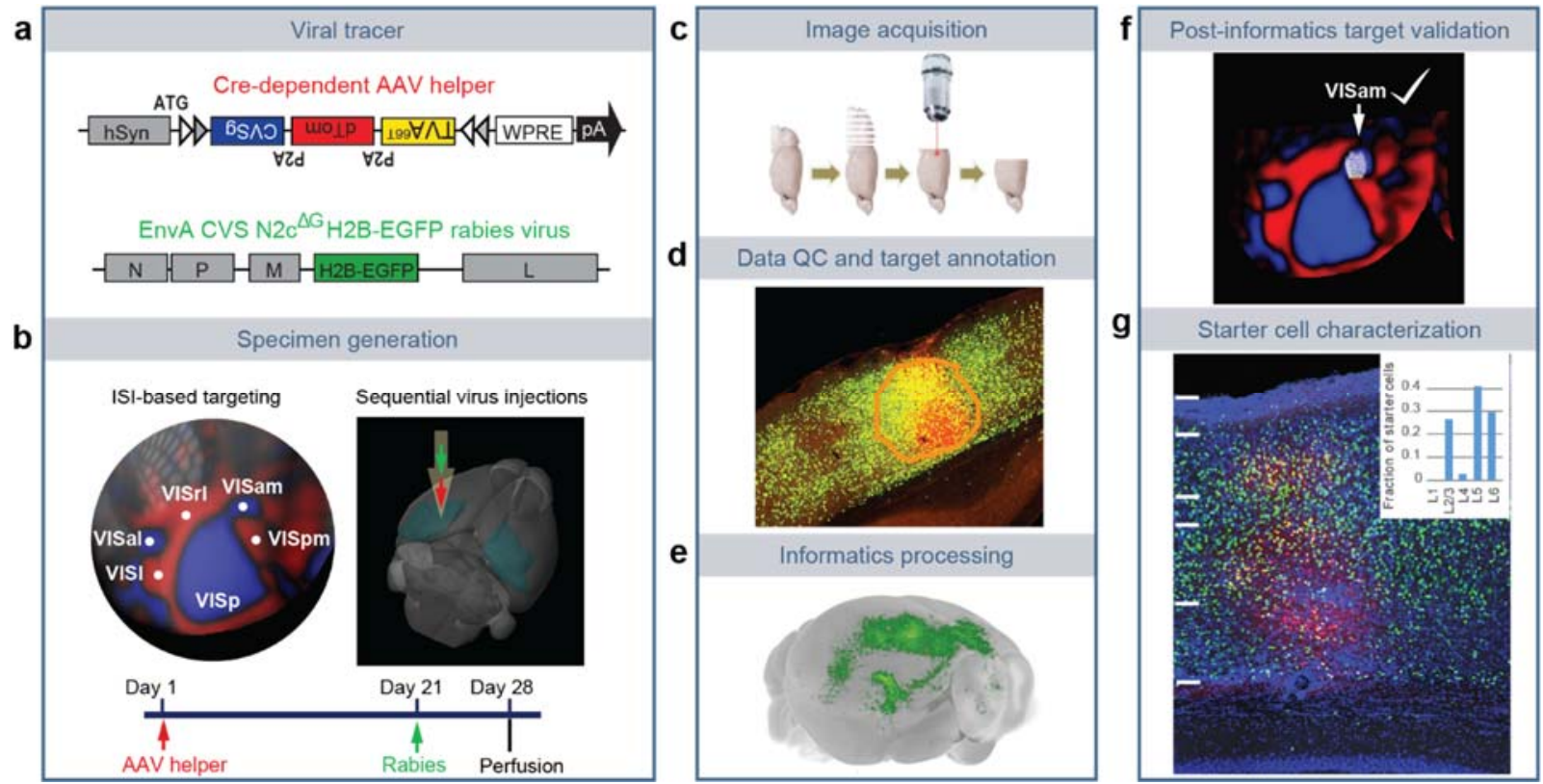

Figure 1. Pipeline identifying monosynaptic inputs to specific neuronal populations in the visual cortex. (a) Viral tools for mapping monosynaptic inputs to Cre-expressing neurons. The tricistronic AAV helper virus conditionally expresses $\mathrm{TVA}^{66 \mathrm{~T}}$, dTomato, and rabies glycoprotein of the CVS N2c strain (CVSg) after Cre-mediated recombination. The EnvA-pseudotyped CVS $\mathrm{N} 2 \mathrm{c}^{\mathrm{dG}}$ rabies virus expresses histone-EGFP (H2B-EGFP) from the rabies $\mathrm{G}$ gene locus in the recombinant rabies virus genome. (b) ISI-based targeting and experimental timeline for virus injections and data analysis. (c) Sequential two-photon images were acquired at $100 \mu \mathrm{m}$ interval and a total of 140 images were obtained for each brain. (d) Target sites were annotated by drawing injection polygons based on the expression of dTomato from the AAV helper virus. (e) Image series was automatically segmented and registered into the Allen CCFv3. (f) Injection site was verified post hoc by overlaying the injection site detected by AAV helper expression with an ISI image. (g) Starter cell characterization. Sections around the planned injection site were collected and dTomato signal was enhanced by immunostaining. Starter cells were then detected by coexpression of dTomato and nuclear EGFP, and the layer-distribution of the starter cells was analyzed.

\section{Comprehensive mapping of inputs to visual areas by cell types}

We utilized our retrograde connectome pipeline to systematically map the presynaptic inputs of neurons in the visual areas by layers and cell classes. A total of 249 experiments across nine excitatory neuron Cre lines and five interneuron Cre lines were included in this study (Figure 2a, and Supplementary Tables 2-3). The Cre lines included those previously used in the Allen Mouse Connectivity Atlas to identify the organization of cortical connections in the mouse brain ${ }^{49}$. 
211 The nine excitatory neuron lines used were the pan-layer Emx1-IRES-Cre, L2/3 IT (Sepw1212 Cre_NP39), L2/3/4 IT (Cux2-IRES-Cre), L4 IT (Nr5a1-Cre), L5 IT (Tlx3-Cre_PL56), L5 ET 213 (A93-Tg1-Cre), L5 IT ET (Rbp4-Cre_KL100), L6 CT (Ntsr1-Cre_GN220), and L6b (Ctgf-2A$214 \mathrm{dgCre})$. The five interneuron lines used were Gad2-IRES-Cre, Ndnf-IRES2-dgCre, Pvalb-IRES215 Cre, Sst-IRES-Cre, and Vip-IRES-Cre. All injections were performed into the left hemisphere, 216 and injection site was verified post-hoc (Methods). We found that all but one experiment (which 217 targeted the temporal association area, TEa), successfully targeted the visual areas (jointly labeled 218 as VIS), including primary visual cortex (VISp), and higher visual areas (HVAs) such as lateral 219 visual area (VIS1, "LM"), posteromedial visual area (VISpm, "PM"), anteromedial visual area 220 (VISam, "AM"), anterior area (VISa, "A"), anterolateral area (VISal, "AL"), rostrolateral visual 221 area (VISrl, "RL"), and laterointermediate area (VISli, "Li"). Fourteen experiments targeted a 222 subarea of the primary somatosensory area barrel field (SSp-bfd) bordering VISrl, which 223 corresponds to previously defined VISrll region and displays extension of retinotopic organization 224 lateral to $\mathrm{VISrl}^{54}$, and thus we refer to this barrel field subarea as SSp-bfd-rll. Locations of all 225 injection centroids were plotted onto the top-down-view of the CCFv3 cortical map (Figure 2b). 226 We quantified presynaptic inputs in each brain area using an automated image 227 segmentation algorithm trained to detect the fluorescence signal from nucleus-localized H2B$228 \mathrm{EGFP}^{52}$. We validated the accuracy of our automatic signal detection and quantification of inputs 229 following registration to the $\mathrm{CCFv} 3$ by comparing the informatically measured per structure input 230 signal volume (sum of detected signal in $\mathrm{mm}^{3}$ ) with manual counting of labeled cells 231 (Supplementary Figure 3a-g). In the six structures from the cortex, thalamus, and cortical 232 subplate, strong positive linear correlations were found between automatic measurement and 233 manual counts ( $\mathrm{R}^{2}$ in the 0.62-0.98 range). Therefore, for subsequent analysis, we used the 234 automatically calculated input signal volume for each structure. 
a

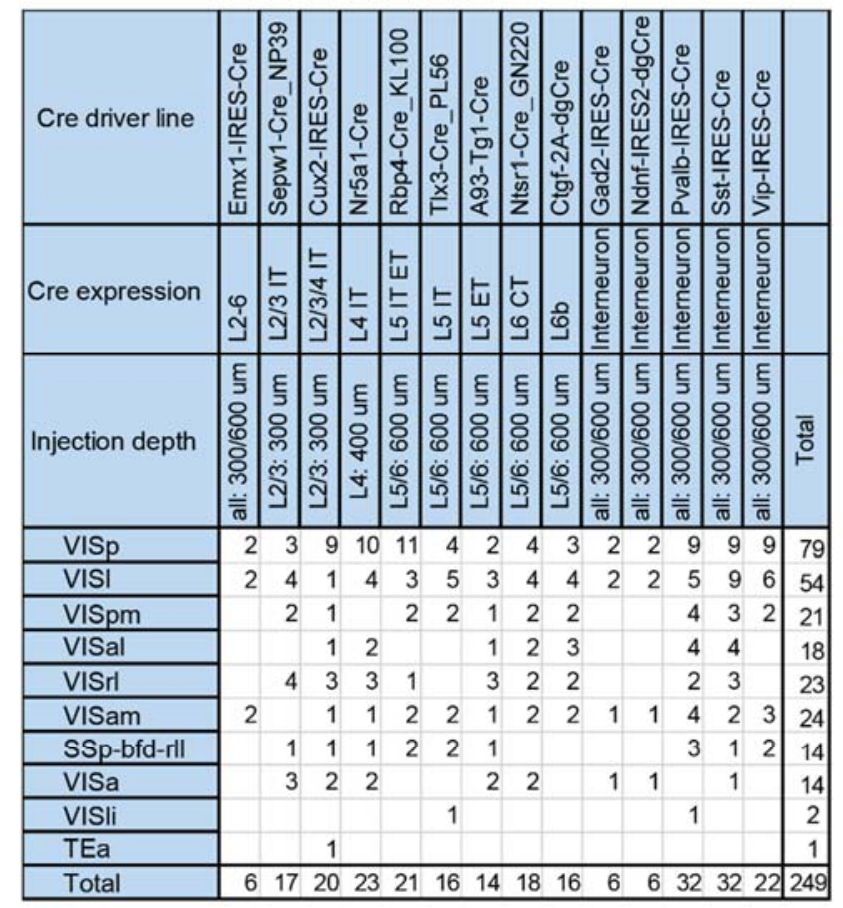

b

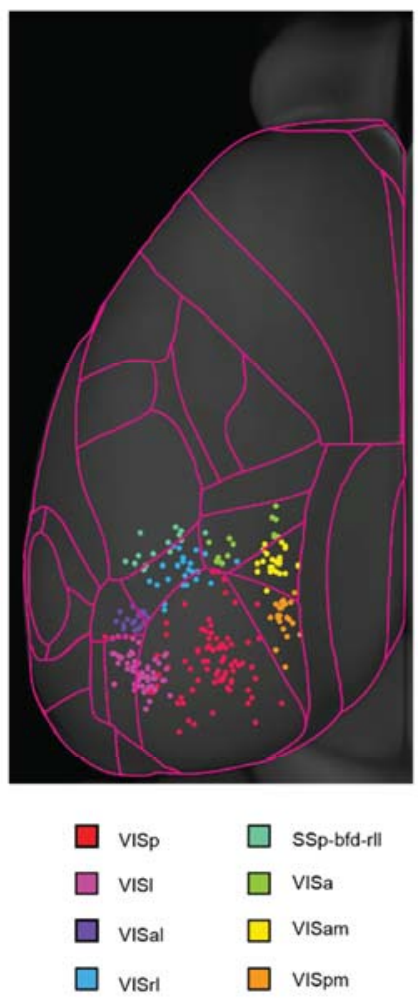

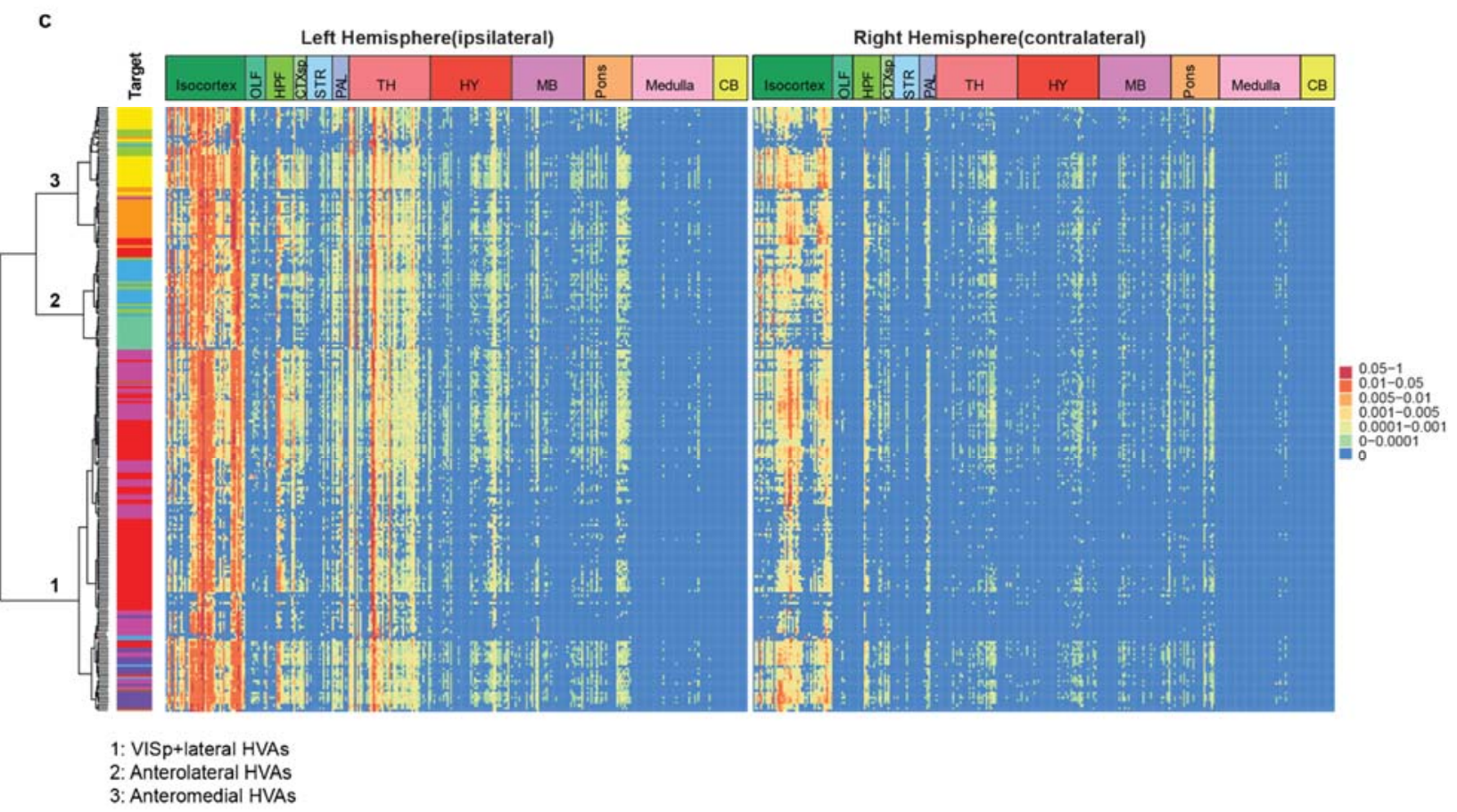

237 Figure 2. Identification of monosynaptic inputs to Cre-labeled neuronal classes in different

238 visual areas. (a) Summary of Cre mouse lines, target areas, and numbers of the 249 experiments 239 in the visual areas. The injection target areas were verified based on overlay of injection site 
polygons with ISI images or the position of injection site polygons in the Allen CCFv3. (b) Mapping of each injection centroid in the top-down view of mouse cortex. Color indicates different visual areas. Red: VISp; Magenta: VISl; Purple, VISal; Light blue: VISrl; Aquamarine: SSp-bfdRLL; Light green: VISa, Yellow: VISam; Orange: VISpm. (c) Matrix showing normalized inputs from the ipsilateral (left) hemisphere and the contralateral (right) hemisphere for the 249 experiments. Each row represents one of the 249 experiments; columns are ordered by 12 major brain divisions; rows are organized according to hierarchical clustering of the input patterns. The input signal per structure was measured by the informatics data pipeline and represented by per structure input signal volume (sum of detected signal in $\mathrm{mm}^{3}$ ). To reduce false positive signal, we identified a set of 92 negative brains that were processed through the pipeline, but showed no rabies-mediated GFP expression, and used this negative dataset to calculate the threshold of false positive signal, i.e., the value of mean input signal volume plus 6 standard deviations for each of the 314 ipsilateral and 314 contralateral major structures of the brain. Input signal not passing this threshold was set to " 0 ". A manually validated binary mask was then applied to remove artifacts in informatically-derived measurements of input signal. Following these two steps, input signal volume of a given structure was normalized to the total input of the brain. Value in each cell of the matrix represents the input signal volume in the given brain area as the fraction of total input of the brain. Color in the "Target" represents the verified injection target area of the experiment in each row, as color-coded in (b).

We find that expression of dTomato from the AAV helper virus faithfully reflects the presence of Cre recombinase. The starter cells show distinct layer-specific distribution patterns consistent with the Cre expression of the respective transgenic lines. The numbers of starter neurons vary between Cre lines and between different experiments within the same Cre line (Supplementary Figure 3h-i, and Supplementary Table 2). Although the overall presynaptic labeling signal increases with the number of starter cells, there is not a strong linear correlation $\left(\mathrm{R}^{2}=0.54\right)$ between the number of starter cells and total input signal volume within the brain (Supplementary Figure 3j). It suggests that postsynaptic cells may receive convergent input from presynaptic cells, which in turn can make divergent connections to different postsynaptic cells. Previously, we compared the whole-brain projections across animals by normalizing the projection signals to the size of the infection area ${ }^{49}$. Here, due to the lack of strong linear correlation between

271 whole brain input signal and the number of starter cells in the injection site, we instead use the

272 fraction of whole brain inputs as our measure of connectivity strength per region, i.e., the input 273 signal volume per brain structure divided by the input signal volume of the entire brain.

274 We next constructed a brain-wide matrix for inputs to the visual areas, focusing on the 275 fraction of whole brain inputs from 314 major structures at a mid-ontology level from the CCFv3 276 (Figure 2c, and Supplementary Table 3). At this anatomical level, hierarchical clustering of the 
277 fraction of whole brain inputs measured from the 249 experiments separate all experiments into 278 three major clusters that are correlated with the spatial proximity of the injection sites: the VISp and lateral HVA cluster (VISl and VISal), the anterolateral cluster (SSp-bfd-rll, and VISrl), and

280 the anteromedial cluster (VISam, VISpm, and VISa). We find that compared to the brain-wide 281 output projections from mouse visual areas $^{49}$, presynaptic inputs come from a broader collection 282 of brain areas (Figure 2c). Visual areas receive the strongest inputs from isocortex (fraction of 283 inputs: median: 0.82 , range: $0.52-0.91$ ), followed by thalamus (median: 0.13 , range: $0.06-0.46$ ) 284 and hippocampal formation (HPF) (median: 0.02, range: 0-0.16) (Supplementary Figure 3k).

\section{Brain-wide inputs to the VISp and HVAs}

Given the correlation between whole brain input patterns and spatial proximity of the injection sites, we compared the presynaptic inputs across all experiments in VISp,VISl, VISal, VISrl, VISa, VISpm, VISam and SSp-bfd-rll. This was possible due to the use of precise injections guided by ISI and sufficient coverage and numbers of injections for all visual areas. Comparison of bilateral inputs from the whole brain (isocortical modules ${ }^{49}$, olfactory area (OLF), HPF, cortical subplate (CTXsp), striatum (STR), pallidum (PAL), thalamus (TH), hypothalamus (HY), midbrain (MB), pons, medulla (MY), and cerebellum (CB)) to the eight target areas reveal overall similar, but quantitatively different global input patterns to different targets, with dominant inputs from the isocortical modules and thalamus (Figure 3a). Visual areas also receive strong presynaptic inputs from HPF, which are mainly found in lateral entorhinal cortex (ENTl), medial entorhinal cortex (ENTm), CA1 and the post-, pre- and parasubiculum (POST, PRE, PAR) (Supplementary Figure 4). In primate, the hippocampal complex and entorhinal cortex are placed at the top of the visual area hierarchy ${ }^{58}$. The entorhinal cortex serves as the interface for a multi-synaptic pathway connecting the visual area with the hippocampus, in which ENTl conveys ventral-stream input to the hippocampus and ENTm conveys dorsal-pathway input ${ }^{65,66}$. Our results reveal direct entorhinal and CA1 inputs to the visual cortex, indicating a visual cortical-hippocampal-visual cortical loop of information processing. A bias for ventral CA1 input to the visual area is also observed, consistent with the distinct projection pathways from the ventral and dorsal CA1 ${ }^{67}$.

Inputs from other anatomical structures each provide less than $1 \%$ of whole brain inputs, and the fraction of inputs span more than three orders of magnitude, ranging from claustrum (CLA) 
308 hypothalamic area (LHA) in HY each representing $\sim 0.1 \%$ of whole brain inputs, globus pallidus, 309 external segment (GPe) in PAL, basolateral amygdalar nucleus (BLA) in CTXsp and zona incerta 310 (ZI) in HY each accounting for $\sim 0.01 \%$ of whole brain inputs, dorsal peduncular area (DP) in OLF, 311 locus ceruleus (LC) in pons, and superior colliculus (SC) in MB each accounting for $\sim 0.001 \%$ of 312 whole brain inputs, to areas in MY each accounting for $\sim 0.0001 \%$ of whole brain inputs 313 (Supplementary Figure 4). CLA is reciprocally connected to various sensory-related brain areas ${ }^{68}$, 314 and the observed strong CLA input to the visual cortex suggests a possible role of CLA in 315 integrating visual processing with other sensory cues. Sparse SC inputs are found in only $11 \%$ of 316 all experiments (Supplementary Table 3) in accordance with the major relay of SC visual input 317 via the lateral posterior nucleus (LP) of the thalamus. Rare inputs in several structures of MY are 318 also found in less than 10\% of all experiments (Supplementary Table 3), which could be missed 319 using other connectivity mapping techniques. 
a

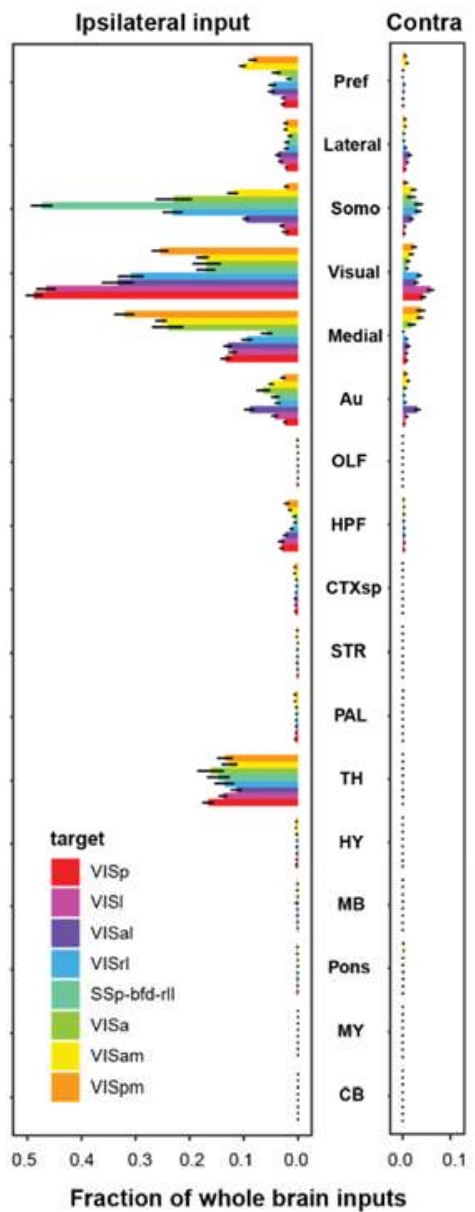

g

- Between different lines in the same target - Between different targets in the same line

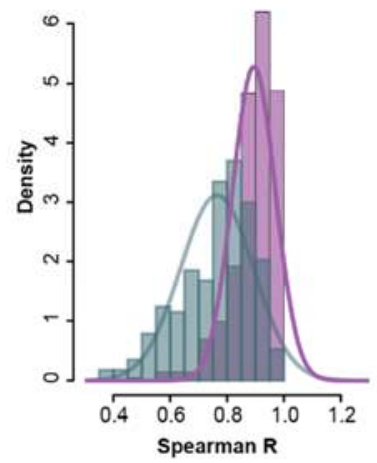

b
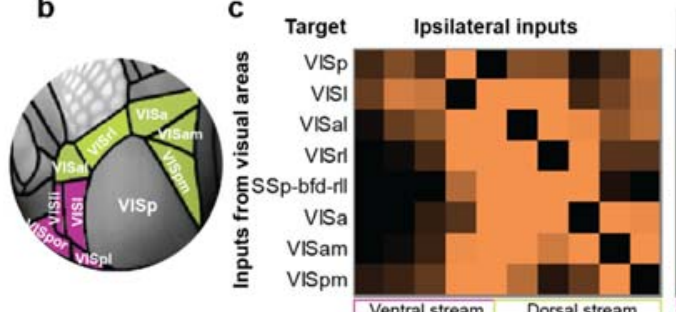

Ventral stream Dorsal stream Ventral stream Dorsal stream

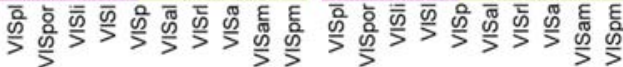
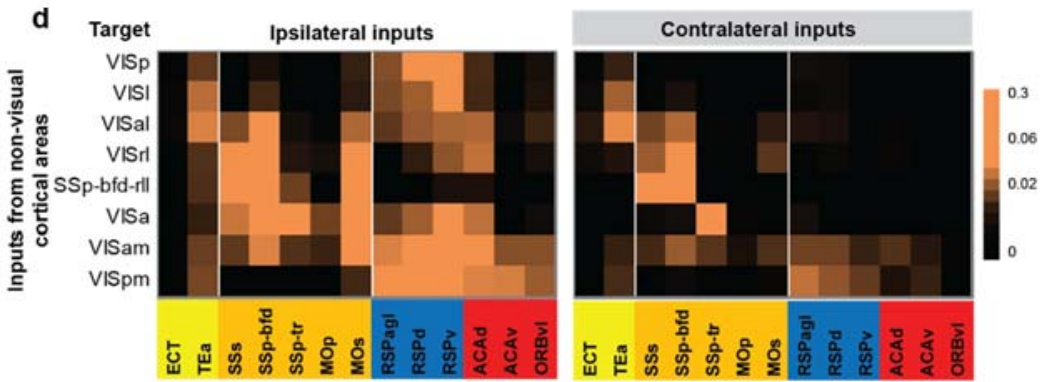

e

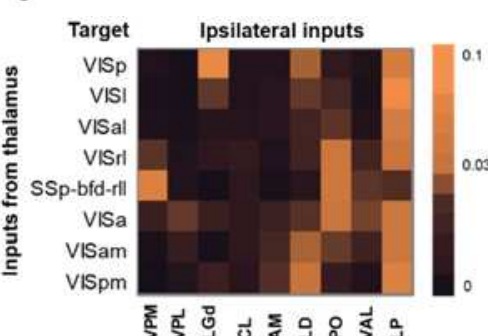

$\mathbf{f}$

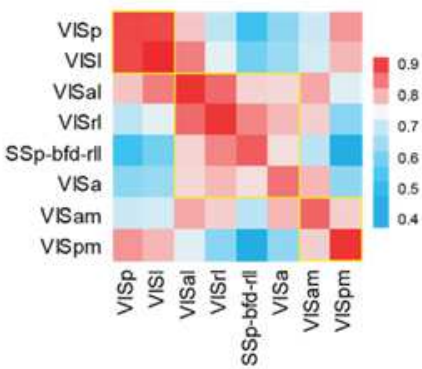

h

Target: VISrI Target: VISpm

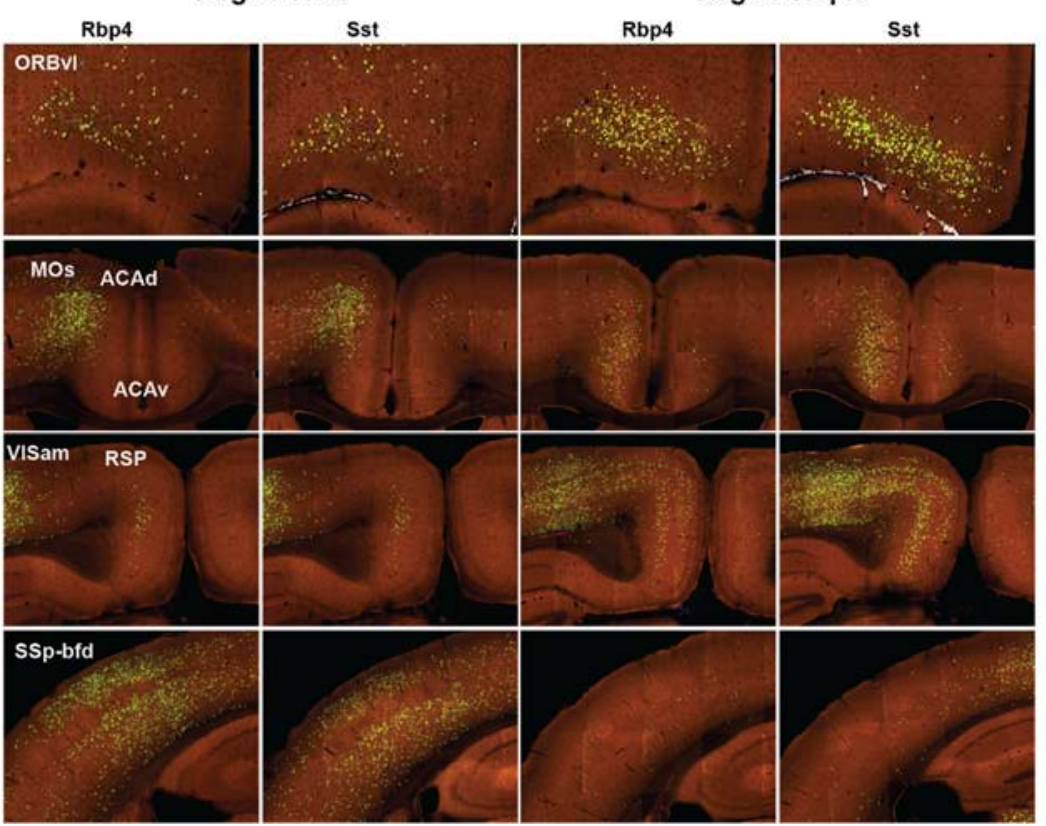



higher visual areas. (a) Comparison of inputs from the isocortex, olfactory area (OLF), hippocampal formation (HPF), cortical subplate (CTXsp), striatum (STR), pallidum (PAL), thalamus (TH), hypothalamus (HY), midbrain (MB), pons, medulla (MY), and cerebellum (CB) to the visual areas. Inputs from the isocortex were divided into six modules: prefrontal (Pref), lateral, somatomotor (Somo), visual, medial, and auditory ( $\mathrm{Au}$ ) (data shown as mean \pm s.e.m.). (b) Visual areas in the ventral and dorsal streams in the top-down view of the CCFv3 cortical map. (ce) Matrices showing the inputs to visual area targets received from within the visual cortex (c), non-visual isocortical modules (d), and thalamus (e). Each row represents the average per structure normalized inputs for experiments with starter cells confined to the same target area, and each cell within the matrix represents the mean value of normalized inputs from a given source area. Visual areas are separated into the dorsal and ventral streams and higher visual areas are in a clockwise order from VISp. Cortical areas with noticeable inputs to visual areas are selected and ordered by module membership. Thalamic areas with noticeable inputs to visual areas are selected and ordered by hierarchical orders. Areas in the thalamus, sensory-motor cortex related, are highlighted in pink and areas in the thalamus, polymodal association cortex related, are highlighted in blue. (f) Matrix showing Spearman's correlation coefficients (R) between experiments within the same target (intra-target) and between experiments across different targets (inter-target) using the input dataset in c-e. Areas with high correlations are highlighted in yellow boxes. The intra-target correlation was calculated as the mean of the spearman correlation coefficients between experiments from different Cre-defined cell types, while the inter-target correlation was calculated as the mean of the spearman correlation coefficients between experiments of the same Cre-defined cell types in different targets. (g) Density plot of Spearman's R for intra-target experiments of different cell types and inter-target experiments of same cell types using the input dataset in b-d. The mean of the intra-target Rs is greater than the inter-target Rs, suggesting that experiments of different cell types in the same target are highly similar to each other. (h-i) Comparison of retrograde input patterns between injections in VISrl and VISpm of Rbp4-Cre and Sst-Cre lines. Rbp4 and Sst experiments in VISrl both show strong inputs from ACAd and SSp-bfd, and weak inputs from RSP and VISam. In contrast, Rbp4 and Sst experiments in VISpm show strong inputs from ACAv, RSP, ORBvl, and VISam, and weak inputs from ACAd and SSp-bfd.

The distribution of subcortical inputs strongly suggests the involvement of neuromodulatory systems in regulating the mouse visual cortex. Clustered inputs are found in NDB (fraction of whole brain inputs in NDB $>0$ in $93 \%$ of all experiments), and substantia innominata (SI) (fraction of whole brain inputs in SI $>0$ in $75 \%$ of all experiments) (Supplementary Figure 4 and Supplementary Table 3), consistent with the innervation of the visual cortex by forebrain cholinergic neurons ${ }^{69,70}$. LC primarily consists of noradrenergic neurons, which send outputs to broad regions of the brain. Our results reveal sparse LC inputs to both VISp and HVAs (fraction of whole brain inputs in LC $>0$ in $66 \%$ of all experiments) (Supplementary Figure 4 and Supplementary Table 3), suggesting that the visual cortex is part of the ascending LC efferent pathway innervating the limbic system, midbrain, thalamus, basal forebrain and 
neocortex ${ }^{71,72}$. In the dorsal raphe (DR) where dorsal cortex-projecting serotonin neurons were

363 previously identified ${ }^{73}$, sparsely labeled presynaptic neurons ( $\sim 0.01 \%$ of whole brain inputs) are

364 found in $63 \%$ of all experiments (Supplementary Figure 4 and Supplementary Table 3).

365 Identification of potential monosynaptic projections from neuromodulator-expressing neurons to

366 both VISp and HVAs suggests that locally released neuromodulators can affect all levels of visual

367 processing.

368 We then focused on individual cortical and thalamic source areas and used the average

369 from all experiments (including all Cre lines) in the same target to compare the input strength from

370 a given presynaptic brain structure to the eight visual targets (Figure 3b-e). The input patterns

371 strongly suggest the presence of two subnetworks equivalent to the dorsal and ventral cortical

372 streams in primates ${ }^{74}$. The nodes in the dorsal stream and the ventral stream of the mouse visual

373 system were previously mapped based on the anterograde projection strength, with VISl, VISli,

374 VISpl, and VISpor in the ventral stream and VISal, VISrl, VISa, VISpm and VISam in the dorsal

375 stream $^{57}$. Our study covers VISal, VISrl, VISa, VISpm and VISam in the dorsal stream, which

376 tends to receive more inputs from each other and fewer inputs from ventral stream structures such

377 as VISpl, VISpor, and VISli (Figure 3c). Consistent with a strong correlation between input

378 patterns and starter cell locations, we find that two adjacent areas, SSp-bfd-rll and VISrl, show

379 similar input patterns characteristic of dorsal stream structures. Both receive strong inputs from

380 the somatomotor cortex, including the secondary somatosensory cortex (SSs), primary

381 somatosensory area barrel field (SSp-bfd), and secondary motor (MOs), but SSp-bfd-rll receives

382 fewer inputs from the midline cortical areas such as anterior cingulate area (ACA) and retrosplenial

383 (RSP) as compared to VISrl (Figure 3d). Given the input patterns of SSp-bfd-rll, we place SSp-

384 bfd-rll as part of the dorsal stream pathway. The ventral stream node, VISl, in general presents

385 similar input pattern as VISp. However, VISp can be distinguished from VISl and other HVAs

386 based on the preference for inputs from the dorsal lateral geniculate complex (LGd) of the thalamus 387 over LP (Figure 3e).

388 Within the dorsal stream, we also find that anterolateral structures such as VISal, VISrl, 389 and SSp-bfd-rll present strong inputs among themselves, while receiving relatively few inputs 390 from the medial structures such as VISam and VISpm (Figure 3c). In contrast, VISam and VISpm 391 show strong mutual connections, while VISpm receives relatively few inputs from the anterolateral 392 structures (Figure 3c). In addition, comparison between inputs to the anterolateral and medial 
structures reveals that the anterolateral structures of the dorsal stream receive stronger inputs from

394 the somatomotor module, whereas the medial structures, VISam and VISpm receive stronger 395 inputs from RSP, ACA and ventrolateral orbital area (ORBvl) (Figure 3d). The anterolateral and 396 medial structures of the dorsal stream also present distinct thalamic input patterns, with VISam 397 and VISpm receiving strong inputs from the anteromedial nucleus (AM) and lateral dorsal nucleus 398 (LD) and anterolateral structures receiving strong inputs from the posterior complex (PO) and 399 ventral anterior-lateral complex (VAL) (Figure 3e). Such input patterns can be observed in both 400 excitatory neuron and interneuron experiments (Supplementary Figure 5). To evaluate the 401 similarity of input patterns between different targets, we calculated Spearman's correlation 402 coefficients $(\mathrm{R})$ for experiments within the same target and for experiments across different targets 403 (Figure 3f). Our results suggest the possibility of two subnetworks in the dorsal stream, one 404 consisting of medial structures of VISam and VISpm and the other consisting of anterolateral 405 structures of VISal, VISrl, SSp-bfd-rll and VISa, with a gradual transition between the two 406 subnetworks from medial to lateral.

407 Given the unique input pattern to each visual area, we further investigated the effects of 408 different starter cell classes on the input patterns. We find that the correlation between input 409 patterns of different cell classes in the same target is higher than that of the same cell class in 410 different targets (Figure 3g-i). Using experiments in VISrl and VISpm as the example, regardless 411 of the starter cell classes, VISrl receives characteristically stronger inputs from ACAd, MOs, and 412 SSp-bfd than VISpm, whereas VISpm receives stronger inputs from ORBvl, ACAv, and RSP than 413 VISrl. Our results suggest that the presynaptic input patterns, as quantified by our tracing system, 414 are predominantly determined by the spatial location of the starter cells, and that different cell 415 classes in the same target receive similar global input patterns.

\section{Comparison of brain-wide inputs to excitatory neurons in different layers}

418 We next focused on quantifying the brain-wide inputs to different Cre-defined 419 glutamatergic excitatory neuron subclasses within a single target area, VISp. Despite the variation 420 in starter cell numbers and layer distributions (Supplementary Figure 6a,b,d), the overall input 421 patterns for any specific location in VISp are similar between different Cre driver lines 422 (Supplementary Figure 6c,e), with most inputs arising from isocortex, followed by thalamus and 423 HPF. Compared to other layer-specific lines, the L4 line receives significantly more input from 
424 the thalamus $(P<0.001$, Tukey multiple comparisons of means $)$, consistent with the notion that 425 feedforward signal from the visual thalamus is mostly received by L4 neurons in the visual $426 \operatorname{cortex}^{75-77}$.

427 We constructed an input strength matrix for experiments targeting 9 Cre-defined excitatory 428 neuron populations in VISp (Figure 4a and Supplementary Figure 7a), and compared that to the 429 brain-wide output projection matrix (Supplementary Table 4) for the same Cre-defined neuron 430 populations in VISp (Figure 4b and Supplementary Figure 7b). In each hemisphere, 43 cortical 431 structures are organized into six corticocortical connectivity modules: prefrontal, lateral, 432 somatomotor, visual, medial, and auditory, as revealed by projection connectivity in our previous 433 study $^{49}$. We find that VISp receives the strongest input from areas within the visual module, 434 followed by visual areas within the medial module. Outside these areas, VISp excitatory neurons 435 receive the majority of inputs from ACA and ORB in the prefrontal module, TEa and ectorhinal 436 (ECT) areas in the lateral module, SSs, SSp-bfd, and MOs in the somatomotor module, RSP in the 437 medial module, and the auditory module. Within the prefrontal module, significantly more 438 presynaptic neurons to VISp are found in ACAd (dorsal part) than ACAv (ventral part, paired t439 test, $P<0.001$ ) and in ORBvl (ventrolateral part) than ORBm (medial part) and ORBl (lateral part, 440 paired t-test, $P<0.001)$. The striking similarity between the patterns of VISp intracortical input 441 and output reveals the reciprocity of corticocortical connections (Figure 4b).

442 Ten thalamic nuclei with the strongest inputs to VISp are included in the input strength 443 matrix (Figure 4a). Three thalamic nuclei, LGd, LP and LD, collectively account for more than $44470 \%$ of the thalamic inputs. These three nuclei also receive strong VISp projections from the L6 445 CT neurons labeled by the Ntsr1 Cre line as well as L5 ET neurons labeled by Rbp4 and A93 Cre 446 lines (Figure 4b), with L6 CT mainly targeting LGd and L5 ET preferentially targeting LP and 447 LD. The brain-wide monosynaptic input matrix also reveals that VISp receives strong inputs from 448 ENTl, ENTm, PAR, POST, CLA and NDB (Supplementary Figure 7a). 

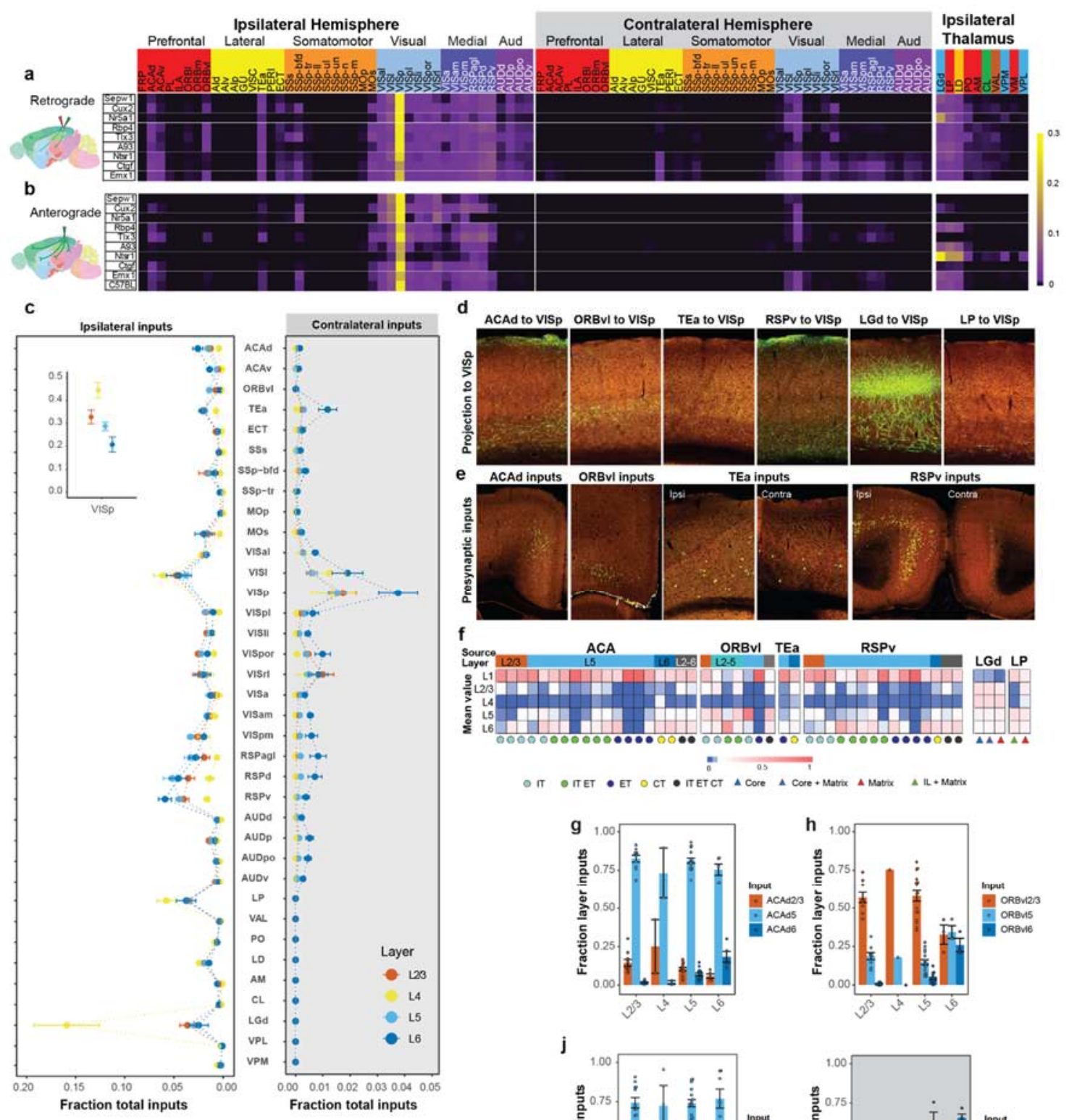

d ACAd to VISp ORBVI to VISp TEa to VISp RSPV to VISp LGd to VISp LP to VISp
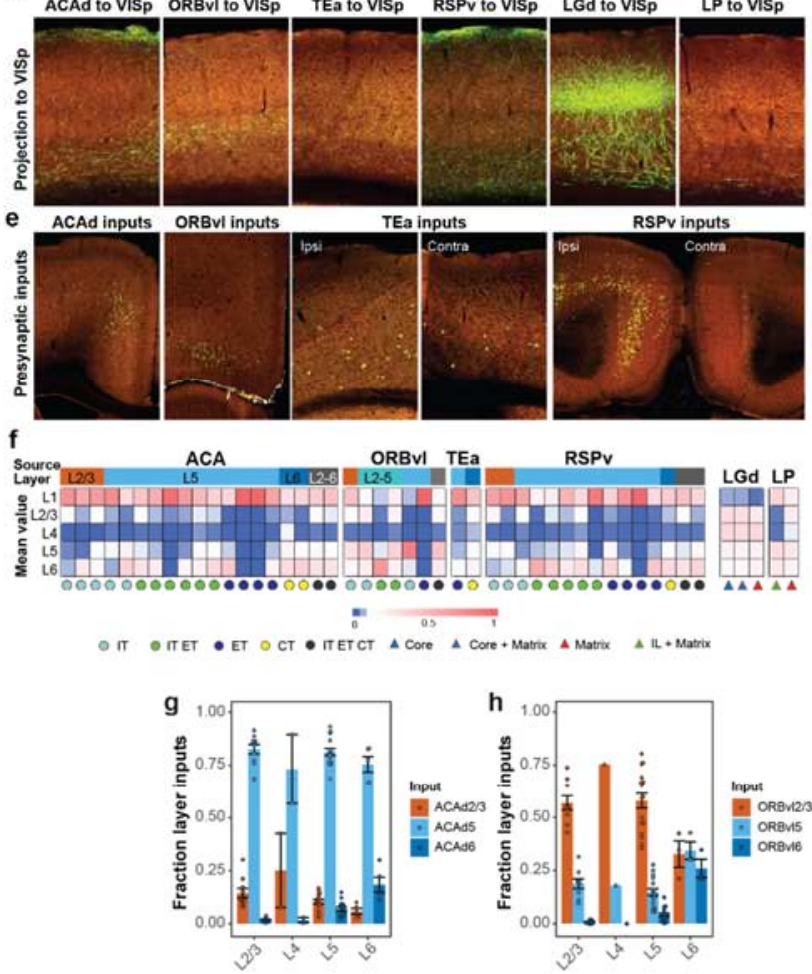

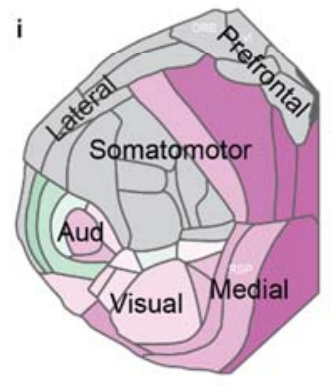

Ipsilateral Hemisphere

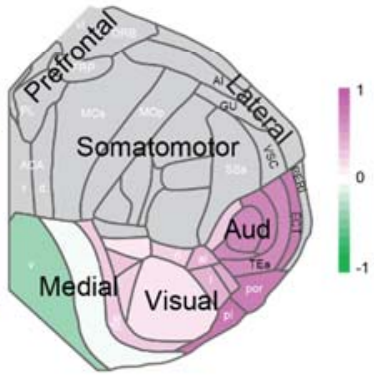

Contralateral Hemisphere
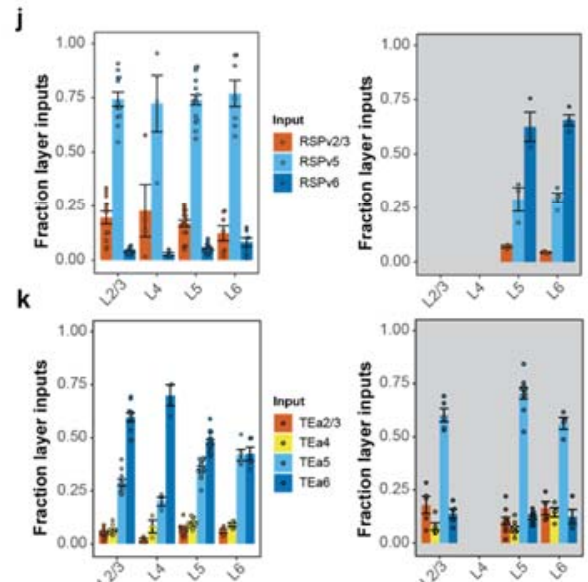

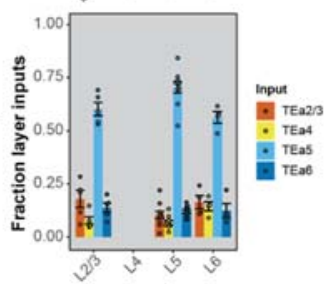

450 Figure 4. Comparison of the whole-brain input patterns to excitatory neuron subclasses in different layers of the primary visual cortex. (a) Matrix showing normalized inputs from the ipsilateral and contralateral isocortex, and ipsilateral thalamus to excitatory neurons in different layers of VISp. Each row of the matrix represents the mean normalized per structure input signals 
for experiments in each Cre line. Rows are organized based on layer-specific distribution of the starter cells. The 43 ipsilateral (left) and 43 contralateral (right) cortical areas are ordered first by module membership (color coded) then by ontology order in the Allen CCFv3. The ten thalamic nuclei are ordered based on the strength of inputs and are colored by the thalamocortical projection classes (blue: core, green: intralaminar, brown: matrix-focal, and red: matrix-multiareal). (b) Matrix showing normalized axonal projections from VISp to the ipsilateral and contralateral isocortex, and ipsilateral thalamus shown in (a). Anterograde tracing experiments (Supplementary Table 4) from the Cre mouse lines used in (a) and C57BL/6J were included, and rows represent the mean normalized per structure projection signals for experiments in each mouse line. (c) Comparison of ipsilateral and contralateral inputs from cortical areas and thalamic nuclei to excitatory neurons in different layers of VISp. Data are shown as mean \pm s.e.m. Representative STPT images showing laminar termination patterns of axon projections in VISp from higher-order association cortical areas and thalamic nuclei. (e) Laminar distribution patterns of presynaptic input cells in higher-order association cortical areas that project to VISp. (f) Normalized laminar termination patterns in VISp for projections from higher-order association cortical areas and thalamic nuclei. Each column represents the relative projection strengths by layer for a unique combination of Cre-defined cell classes and source areas. The average value was taken when $n>1$. L6b was excluded due to low accuracy in informatic quantification of projection signal in L6b. (g-h) Laminar distribution of long-range inputs from ACAd (g) and ORBvl (h) to excitatory neurons in different layers of VISp. The fraction layer input is calculated as the fraction of the total input signal in a given source area across layers. L1 is excluded from the analysis due to overall lack of signal in this layer. The calculated fraction layer inputs are consistent with representative images of inputs in ACAd and ORBvl to VISp (e). Data are shown as mean \pm s.e.m. (i) Comparison of L5 and L6 preference for source cortical areas in the ipsilateral (left) and contralateral (right) hemispheres sending presynaptic inputs to VISp. The preference score for a given cortical area is calculated as (L5 input - L6 input) / (L5 input + L6 input). Each source cortical area was colored according to its preference score. (j-k) Laminar distribution of inputs in RSPv (j) and TEa (k) to VISp as examples of source cortical areas located in the medial (RSPv) or lateral (TEa) areas of the cortex. Data are shown as mean \pm s.e.m.

We then compared the input patterns of excitatory neurons in different layers of VISp

(Figure 4a, c and Supplementary Figure 7a, c). We find that L4 neurons receive the least amount of input from higher-order association cortical areas, including ACA, ORBvl, TEa and RSP, as well as from subcortical input areas, and receive significantly more inputs from LGd. In contrast,

491 the axonal lamination patterns from cortical and thalamic areas to VISp (Figure 4d,f), where

492 ORBvl axons primarily ramify in L1 and L5 of VISp, ACAd axons mainly reside in L1 and L6, 493 and axons from LGd mainly ramify in L4. 
in different layers (Figure 4e, $\mathbf{g - h}$ ). We find that the presynaptic input neurons in higher-order

496 association cortical areas are often located in deep layers (L5 and L6), regardless of the layer 497 location of starter cells in VISp. For example, in ipsilateral ACAd, L5 contains the most 498 presynaptic neurons compared to other layers, with a similar preference for L5 observed for inputs 499 to excitatory neurons located in different layers of VISp (Figure 4e,g). A noticeable exception is 500 ipsilateral ORBvl, in which VISp L2/3, L4 and L5 neurons preferentially receive inputs from L2/3

501 ORBvl whereas no preference in layer distribution is found for input to L6 neurons of VISp 502 (Figure 4e,h).

We also investigated whether inputs from homotypic ipsilateral and contralateral cortical areas to VISp arise from different layers (Figure 4i-k) and which source layer contributes most to VISp. Since most inputs arose from L5 and L6, we calculated a preference score for a given cortical area as (L5 input - L6 input)/(L5 input + L6 input). We find that medial areas of the ipsilateral hemisphere show a bias toward L5 input to VISp, with the preference gradually shifting towards L6 in lateral areas, whereas areas of the contralateral hemisphere present an opposite bias: medial areas show L6 bias, and lateral areas show L5 bias (Figure 4i).

These distinct features in whole-brain input patterns to excitatory neurons in different layers of VISp can also be found in VISl (Supplementary Figure 8 and Supplementary Table

512 5). Similar to our observation in VISp, we find that L4 overall receives more inputs from thalamic 513 areas and fewer inputs from higher-order cortical areas, most inputs from higher-order cortical 514 areas are from the deep layers, and ipsilateral and contralateral cortical areas present different 515 laminar distribution of input neurons to the same target. We also find generally consistent input 516 patterns to excitatory neurons in different layers of other HVAs as of VISp and VISl, though due 517 to smaller number of experiments in each layer of each region (Figure 2a) we do not provide 518 quantitative analysis here.

\section{Distinct presynaptic inputs to L6 excitatory neurons of visual areas}

We observed that L6 CT and L6b neurons (as labeled by Ntsrt1 and Ctgf Cre lines) clearly receive more inputs from the contralateral cortex compared to excitatory neurons in the other layers

523 (Figure 4a and Supplementary Figure 6e). These inputs mostly originate from the contralateral 524 visual, medial, and auditory modules. To further investigate whether the layer distribution of starter neurons is the key factor in determining the level of contralateral inputs, we identified 89 
526 experiments in VISp and HVAs with starter cells restricted to a single layer and compared the 527 contralateral and ipsilateral inputs between the experiments. Overall, L6 neurons across VIS 528 receive more contralateral inputs from all six isocortical modules than neurons in other layers 529 (Supplementary Figure 9). Quantitative analysis suggests that the effect of layer on the ratio of 530 contralateral to ipsilateral isocortical inputs is significant (two-way ANOVA, $P<0.001$ ), and that 531 the location of the target site (VISp or HVAs) does not significantly affect the ratio of contralateral 532 to ipsilateral isocortical inputs $(P=0.37)$. Our results suggest that L6 of the visual area has distinct 533 retrograde connectivity compared to the other layers.

\section{Brain-wide inputs to VISp interneurons}

To explore presynaptic inputs to distinct GABAergic interneurons, we employed various

537 Cre lines driven by genes corresponding to major interneuron subclasses: parvalbumin (Pvalb)538 expressing, somatostatin (Sst)-expressing, vasoactive intestinal peptide (Vip)-expressing neurons, 539 and neuron-derived neurotrophic factor (Ndnf)-expressing neurons which are mostly L1 540 neurogliaform cells. We also included the Gad2-Cre line to cover all cortical interneurons.

541 Despite variation in starter cell numbers (Supplementary Figure 3) and layer distribution, 542 the overall global patterns are again similar between Cre lines, regardless of excitatory or 543 inhibitory type (Figure 5a). We quantified the fraction of inputs from cortical modules and 544 thalamus to excitatory neurons and interneuron cell classes located in VISl, VISp and VISam, 545 where we had experiments covering almost all the 14 Cre-defined cell classes. We find that, 546 compared to excitatory neurons, interneurons overall receive more inputs from thalamus and 547 ipsilateral visual cortical module (Figure 5b), and fewer inputs from contralateral cortical modules 548 (Figure 5b-c), suggesting that intra-module inputs exert greater influence on interneurons than 549 excitatory neurons. 
a

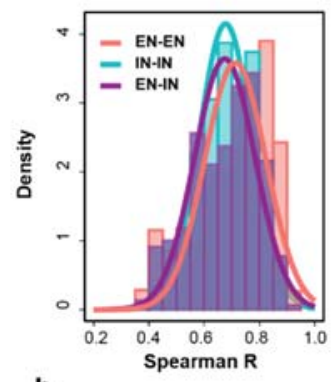

$$
\text { b }
$$
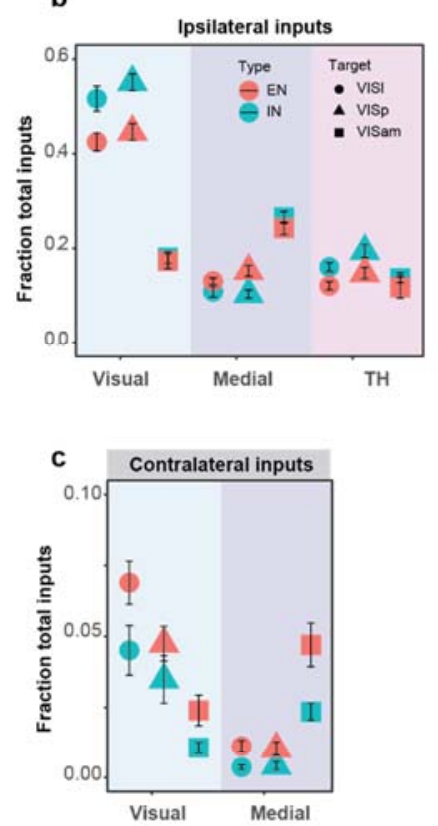

d

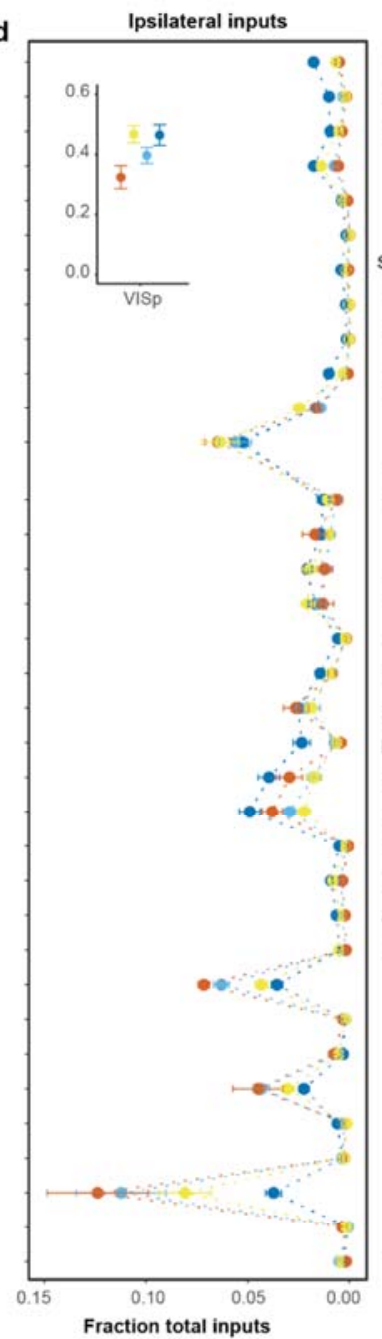

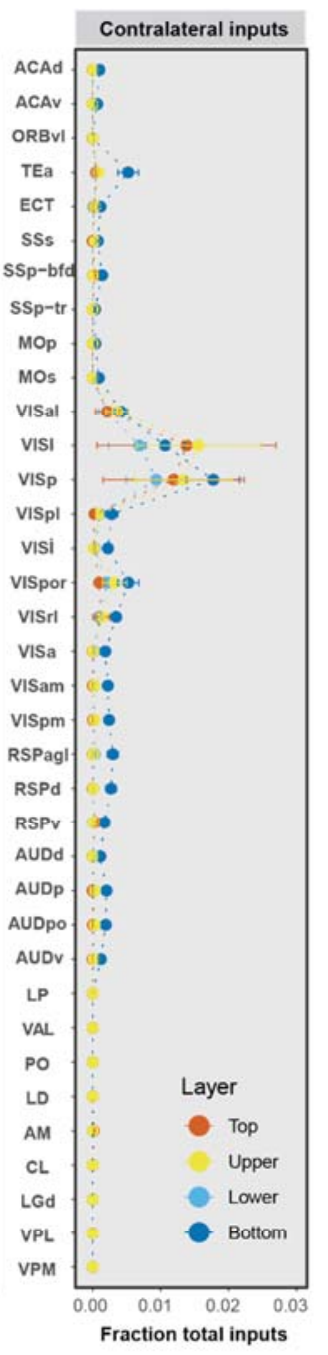

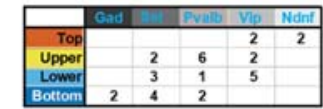

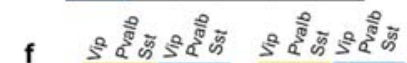

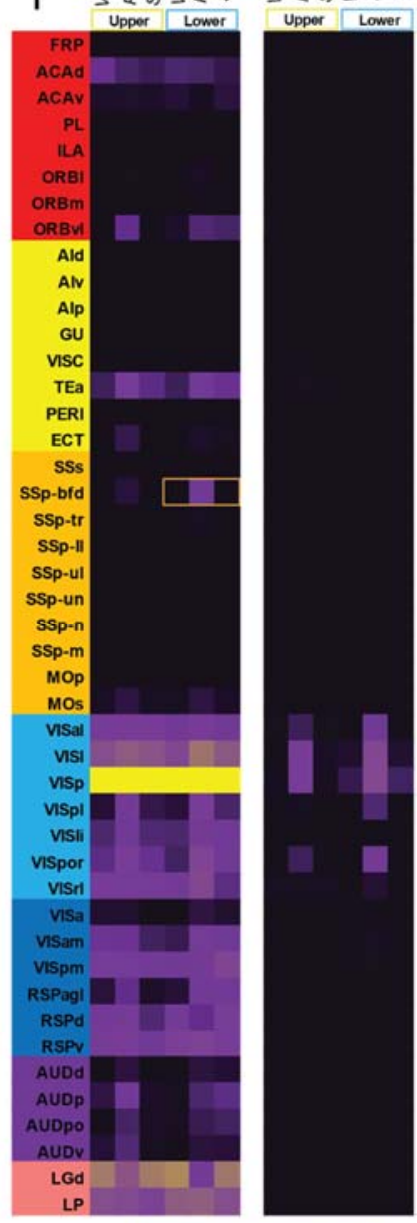

$\begin{array}{lll}0.3 & 0.2 & 0.1\end{array}$

g

Target
area
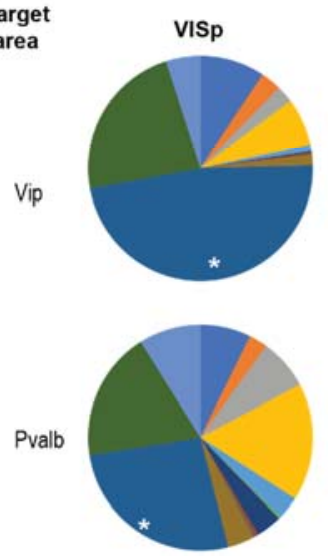
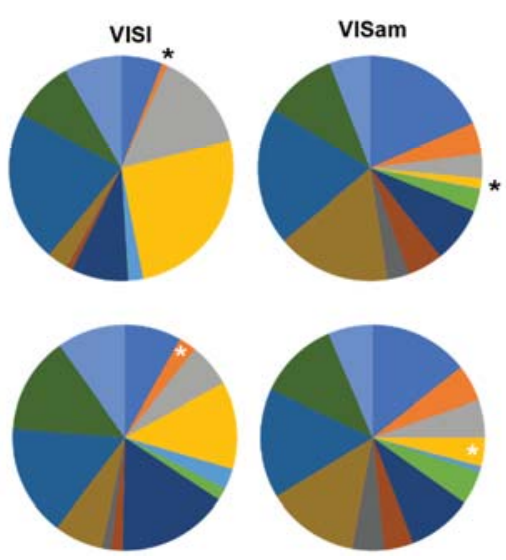

$$
\begin{aligned}
& \text { - ACAd = ACAv = ORBvl } \\
& \text { = TEa }=\mathrm{ECT}=\mathrm{SSs} \\
& \text { - SSp-bfd a SSp-tr a MOp } \\
& \text { - MOS a RSPV a RSPd } \\
& \text { n RPagl }
\end{aligned}
$$

Figure 5. Comparison of the whole-brain input patterns to different interneuron subclasses in the primary visual cortex. (a) Density plot of Spearman's $R$ values for whole-brain input 
subclasses, and Rs measured between EN input patterns and IN input patterns. The means of the inter-cell-class Rs and intra-cell-class Rs are close to each other, suggesting that input patterns to EN and IN classes are highly similar to each other. (b-c) Comparison of ipsilateral (b) and contralateral (c) inputs from the visual and medial cortical modules and thalamus to EN and IN cell classes located in the VIS1 (circle), VISp (triangle), and VISam (square). Data are shown as mean \pm s.e.m. (d) Comparison of inputs from ipsilateral and contralateral cortical areas and thalamic nuclei to interneurons located in different depths of VISp. Data are shown as mean \pm s.e.m. (e) Summary of the depth distribution of IN experiments in VISp. (f) Matrices showing normalized inputs in the ipsilateral cortex, LP and LGd to the three IN subclass experiments in the upper and lower groups. Each column of the matrix represents the mean normalized input signals for the IN subclass. The area with statistically significant difference in inputs to Pvalb and Vip cells is boxed in yellow. (g) Comparison of relative input strength from higher-order cortical areas to Pvalb and Vip cells in VISp, VISl and VISam. Areas with statistically significant differences between the two interneuron subclasses are labeled with asterisks.

We then focused on different interneuron subclasses in VISp to investigate the possibility of cell-class-specific input patterns. To account for the contribution of layer distribution of starter cells to input patterns (Supplementary Figure 10), we divided the 31 interneuron experiments in VISp into four different groups based on the depth of starter cell population: the Top group contained experiments with starter cells restricted to L2/3 (we found very few L1 starter cells), whereas the Upper, Lower, and Bottom groups had progressively more starter cells in deep layers (Supplementary Figure 10b). Although starter cells in these groups are rarely restricted to a single layer, we find distinct input patterns of interneurons, especially between the Bottom group and others. Compared to other groups, the Bottom group receives more inputs from higher-order cortical areas, including the frontal, sensorimotor, and auditory modules, and the fewest inputs from thalamic areas such as LGd and LP (Figure 5d). Consistent with the observation of L6 excitatory neurons receiving extensive contralateral cortical inputs, the Bottom group also receives more contralateral cortical input than the other groups (Figure 5d). We then compared Sst, Vip, and Pvalb experiments in the Upper and Lower groups to avoid the confounding influence of layer distribution of starter cells on input patterns (Figure 5e-f). Despite variations of normalized inputs from higher-order cortical areas between Vip and Pvalb, statistical significance was not observed for most presynaptic areas, likely due to limited sample sizes. The distinct patterns of cortical inputs between Vip and Pvalb can also be observed when comparing all Vip and Pvalb experiments in VISp, VISl, and VISam, regardless of starter cell layer distribution (Figure 5g). Both cell classes present unique target-specific cortical input patterns, and within the same target, Vip and Pvalb also differ in the relative input strength from selected higher-order cortical areas. 
a

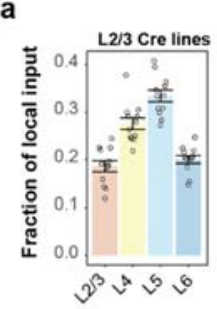

b

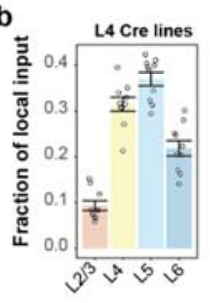

C
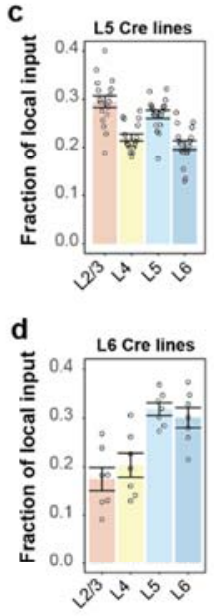
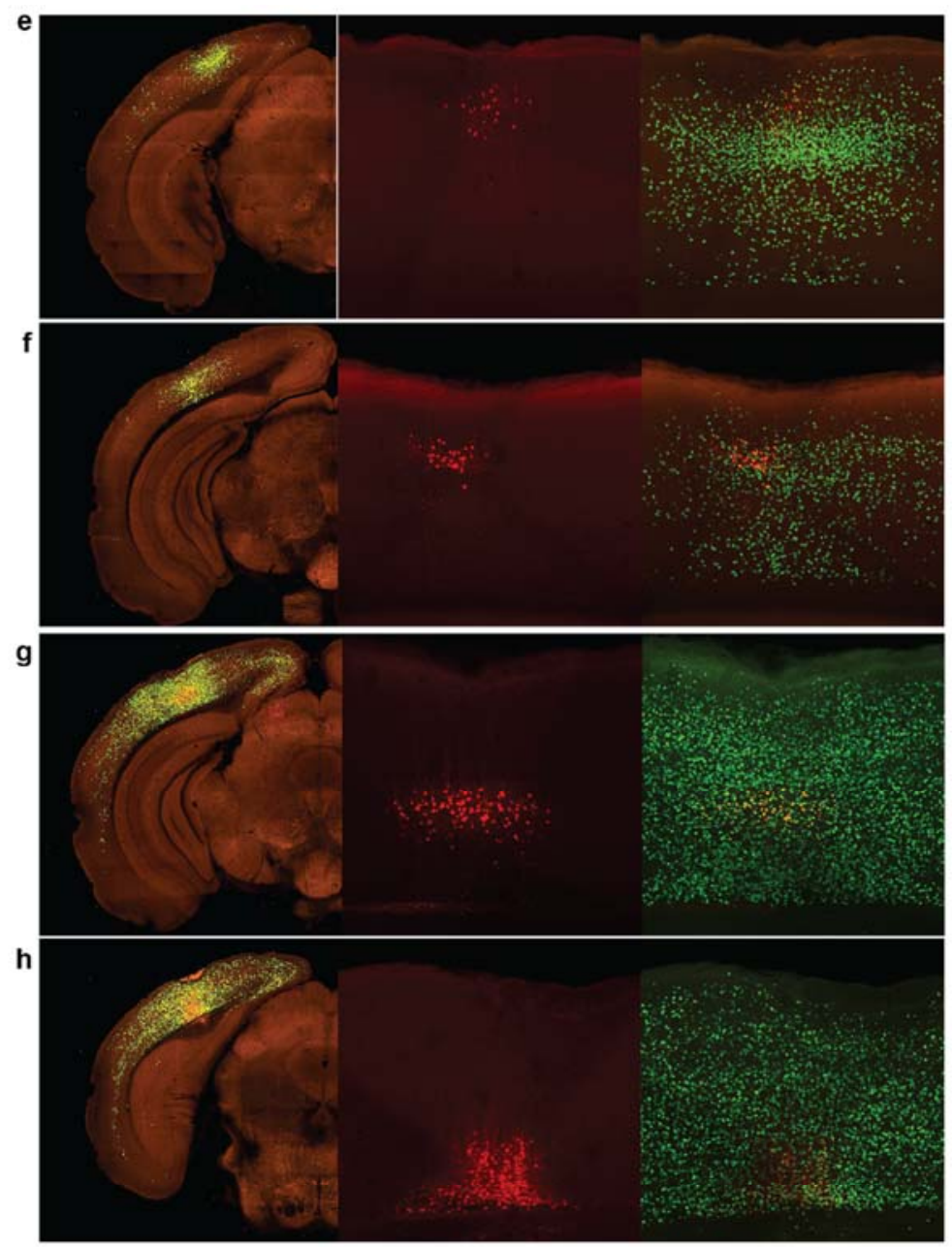

Upper
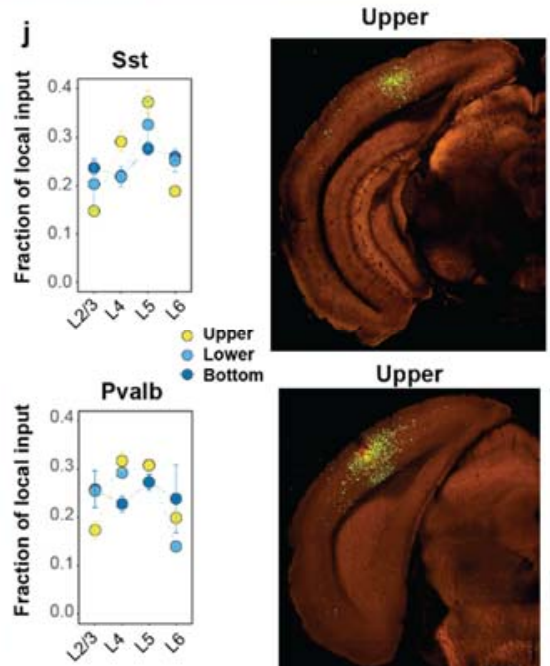

Upper

Bottom

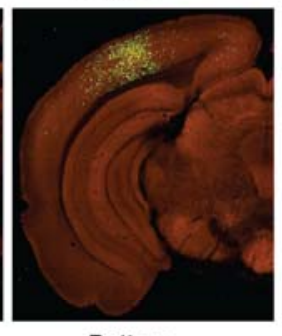

Bottom
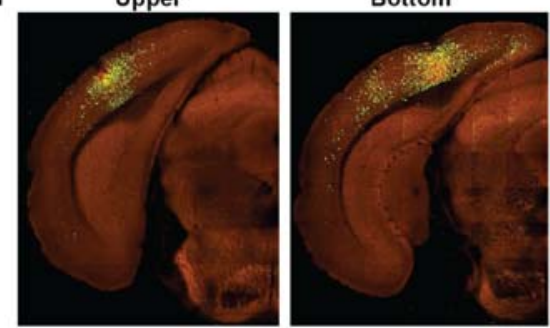
Figure 6. Comparison of local inputs to excitatory neurons and interneurons in different depths of the VISp. (a-d) Fraction layer input of ipsilateral VISp inputs to excitatory neurons in L2/3 (a), L4 (b), L5 (c) and L6 (d) of VISp. Data are shown as mean \pm s.e.m. (e-h) Representative images showing layer-specific local inputs to excitatory neurons in L2/3 (e), L4 (f), L5 (g) and L6 (h) of VISp. The left panels show STPT images of brain sections containing starter cells, and the right two panels are confocal microscopic images showing the distribution of starter cells and local inputs. Starter cells are identified by the coexpression of dTomato from the AAV helper virus and nucleus-localized EGFP from the rabies virus. (i) Comparison of local input patterns to Ndnf-Cre and Vip-Cre experiments with Top distribution of starter cells. Data are shown as mean \pm s.e.m. (j) Comparison of local inputs to Sst and Pvalb experiments with different depths of starter cell distribution. Representative images containing the injection sites are provided for Sst-Cre and Pvalb-Cre experiments in the Upper and Bottom groups. Data are shown as mean \pm s.e.m.

\section{Local inputs to excitatory neurons and interneurons}

In experiments where the starter cells were restricted to a specific layer of VISp or VISl, 611 preference of any local input as the fraction of input in that layer compared to all local inputs. We 612 find that the starter cells in each layer receive characteristic local input patterns. In VISp, L2/3 613 excitatory neurons preferentially receive inputs from L4 and L5. L4 excitatory neurons receive the 614 fewest inputs from L2/3 and preferentially receive inputs from L4 and L5. L5 excitatory cells 615 receive strong inputs from L2/3 to L6, with a noticeable preference for L2/3 input, and L6 616 excitatory neurons preferentially receive inputs from the deeper layers. With the exception of L2/3, 617 starter cells receive dense inputs from other cells in the same layer. Our results support dense 618 inputs from L4 to L2/3, despite weak input from L2/3 to L4, and dense reciprocal inputs between 619 L2/3 and L5. Analysis of the local input patterns to excitatory neurons with layer-specific distribution in VISl also reveals that starter cells in each layer receive characteristic local inputs similar to excitatory neurons in VISp (Supplementary Figure 11).

Considering the layer-specific local input patterns observed in excitatory neurons, we 624 of starter cell distributions (Figure 6i-j). The Top group includes two Ndnf experiments and two 625 Vip experiments, with the Ndnf starter cells receiving the most inputs from L5, and the Vip starter cells receiving strong inputs from L4 and L5. The Upper, Lower, and Bottom groups each exhibit

627 distinct local input patterns, with the Bottom group receiving more inputs from L6 than the Upper 628 group. Our results suggest cell-class- and layer-dependent local input patterns for both excitatory neurons and interneurons. 


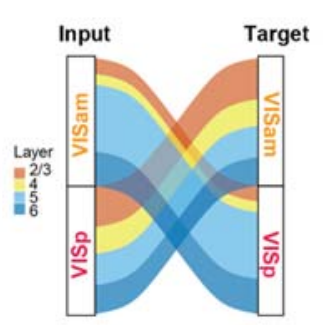

$d_{L 2 / 3 / 4}$

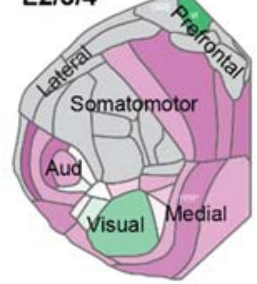

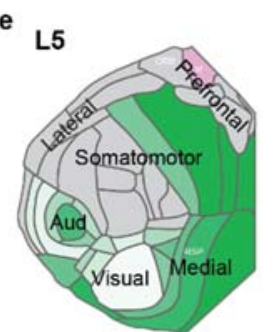

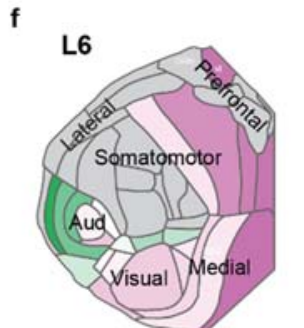

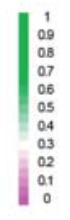

b

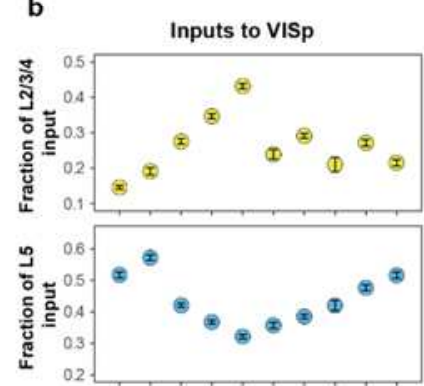

c
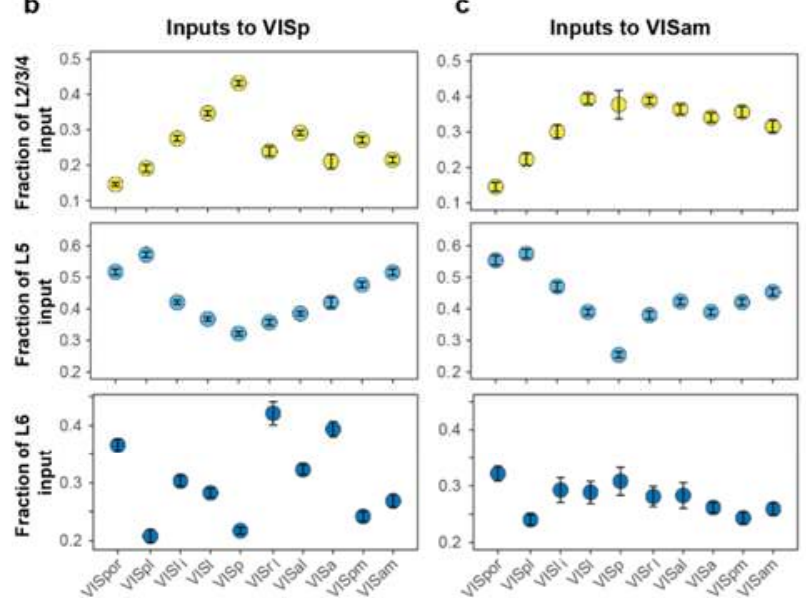

h
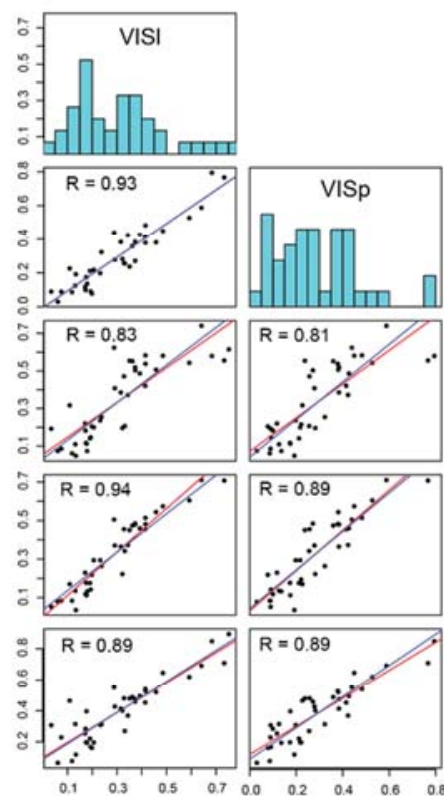

$R=0.89$

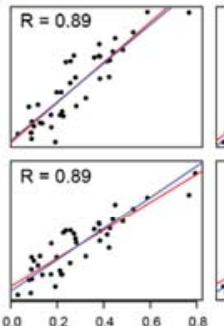

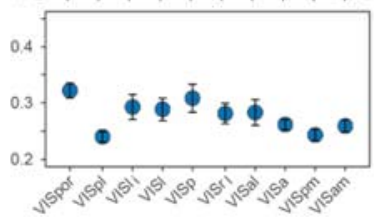

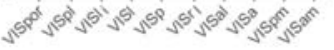
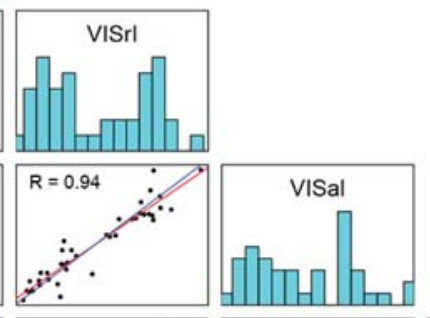

$R=0.86$

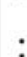

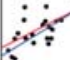

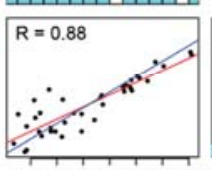

g

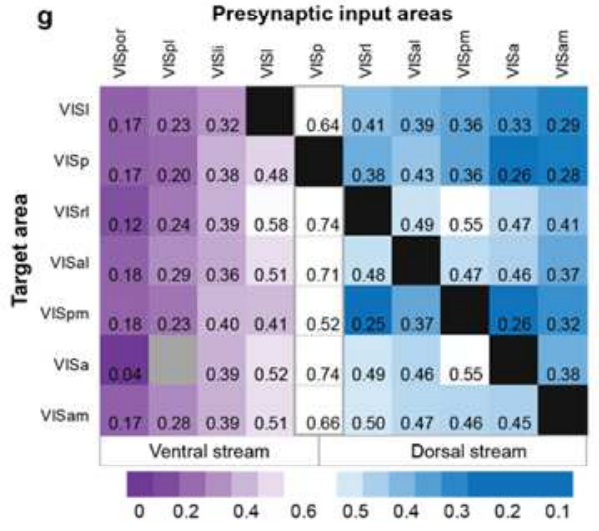

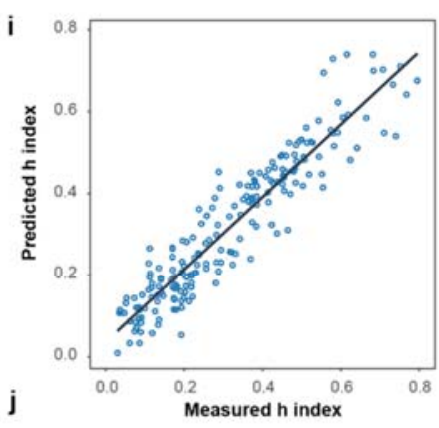

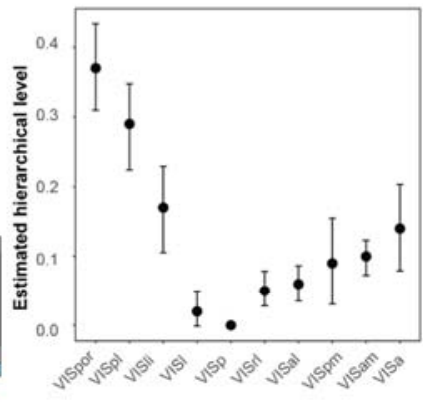


Figure 7. Relative hierarchical positions of the primary visual cortex and higher visual areas. (a) Laminar distribution of inputs for connections between VISp and VISam. Fractions of total inputs in the source area across layers were calculated for each experiment. Mean fraction of layerspecific inputs was used to represent the laminar distribution of inputs between the source area and the target area. (b-c) Comparison of laminar distribution of visual area inputs to VISp (b) and VISam (c). Fractions of total inputs in the source area across layers were calculated for each experiment. Each dot represents the mean ( \pm s.e.m.) fraction of layer-specific input from the source area to the target area. Visual areas are organized based on previously predicted hierarchical positions and separated into the dorsal and ventral streams. (d-f) Comparison of the fraction of L2/3/4 input (d), L5 input (e) and L6 (f) input from various cortical areas to VISp. Mean fraction layer input was calculated to represent the laminar distribution of inputs between the source area and VISp. Each source area is colored according to the mean fraction of inputs in L2/3/4 (d), L5 (e) and L6 (f), respectively. (g) Matrix of $h$ index of inputs from the 10 visual areas to the 7 targets. $h$ index is calculated as the ratio of inputs in layers $2 / 3 / 4$ to the sum of inputs in layers $2 / 3 / 4 / 5$. The visual areas are separated into the ventral and dorsal stream. Each cell within the matrix represents the mean $h$ index value of inputs in a given source area to a target area. Gray cell represents no availability of data due to sparse inputs from the source area. (h) Pairs plots showing the correlation of measured $h$ index values of cortical source areas sending inputs to specific pairs of target areas. Each point represents the average pair of $h$ index values obtained in a given source area to a pair of target areas as indicated at the top and the right of each graph. VISa is not included as one of the target areas due to a low number of experiments in VISa, and VISpm is excluded due to the lack of experiments in L4. The red lines are the best fit lines (least-squares regression lines), and the blue lines are the lines with a slope equal to 1 that best fit the points. (i) Correlation between measured and predicted $h$ index values between cortical source areas and the five target visual areas in panel $\mathrm{h}$. We used the linear regression analysis to estimate a set of hierarchical levels that best predict the measured $h$ values. A model can be specified as $\mathrm{Y}=\mathrm{X} \beta$, wherein $\mathrm{Y}$ is a vector containing the $h$ values of all source areas to each target, $\beta$ contains the estimated hierarchical levels assigned to each area, and $\mathrm{X}$ is the incidence matrix. $\mathrm{X}$ is constructed so that each column

\section{Hierarchical order of mouse visual areas defined by presynaptic inputs}

We subsequently explored whether the laminar distribution of presynaptic inputs to visual areas reveals the hierarchical ordering of these areas. In primates, the hierarchy of visual cortical areas was derived by designating anatomical connections to feedforward and feedback directions, with feedforward connections originating from superficial layers in a lower area and terminating 
674 in L4 in a higher area, and feedback connections originating from deeper layers in a higher area 675 and terminating outside L4 in a lower are ${ }^{58}$. Other studies used the fraction of labeled presynaptic 676 supragranular neurons (SLN), defined as the number of labeled neurons in L2/3 divided by the 677 sum of labeled neurons in supra- and infra-granular layers (L2/3 + L5 + L6), to derive a 678 hierarchical ordering of the primate visual cortex areas that was consistent with the Felleman and 679 Van Essen hierarchy ${ }^{58,59}$. Unlike what was described for primates, L4 neurons in mouse VISp do 680 appear to play an important role as well as other layers in information relay to HVAs. A preference 681 for L4 inputs from VISp is particularly noticeable for VISrl and SSp-bfd-rll, with the highest 682 fraction of VISp inputs coming from L4 (Supplementary Figure 12).

683 In an effort to identify a quantitative hierarchical parameter for visual cortex areas using 684 retrograde labeled cells, we first compared the laminar distribution of presynaptic inputs for the 685 connections between VISp and VISam (Figure 7a). In our previous cell-class-specific axon 686 projection mapping ${ }^{49}$, we predicted that VISp lays at the base of the visual area hierarchy, whereas 687 VISam resides at the top of the hierarchy. Accordingly, inputs from VISam to VISp are considered 688 feedback inputs, whereas those from VISp to VISam are considered feedforward inputs. We find 689 that the percentages of L2/3 and L4 inputs of the VISp-to-VISam connection are significantly 690 higher than those of the VISam-to-VISp connection $(P<0.001$, two sample t-test), and the 691 percentage of L5 inputs from VISam to VISp is significantly higher than that from VISp to VISam $692 \quad(P<0.001$, two sample t-test). In contrast, the percentage of L6 input does not show statistically 693 significant difference between the two directions $(P>0.05$, two sample t-test). Examination of 694 visual area inputs to VISp and VISam further confirmed that the fractions of input from L2/3/4 695 and L5 present a complementary pattern consistent with the predicted relative hierarchical 696 positions of each source area and target area based on laminar projection patterns (Figure 7b-c). 697 We also compared the laminar distribution of inputs from various ipsilateral cortical areas to VISp 698 (Figures 7d-f), and find that inputs from almost all other cortical areas to VISp show lower fraction 699 of L2/3/4 inputs and higher fraction of L5 inputs than intra-VISp inputs, consistent with separate 700 roles of L2/3/4 and L5 in feedforward and feedback information relay. In contrast, the fraction of 701 L6 input shows a lateral to medial gradient with fraction of L6 input higher in the lateral areas and 702 lower in the medial area.

703 Our observations suggest that the ratio of inputs from the superficial L2/3/4 to the sum of 704 inputs from the superficial layers and L5 (hereinafter referred to as the $h$ index) could be used to 
705 quantify the hierarchical positions of the mouse visual areas. We calculated the average $h$ index 706 for presynaptic inputs from 10 source visual areas to 7 target visual areas (Figure $\mathbf{7 g}$ ). For a given 707 target, inputs from VISp exhibit the highest $h$ index values as compared to the HVAs, consistent 708 with VISp at the lowest hierarchical position, and inputs from HVAs of higher hierarchical 709 positions in the ventral and dorsal streams have lower $h$ index values as compared to other HVAs 710 in the same stream.

711 To explore whether $h$ index can serve as a quantitative parameter for hierarchical distance 712 between cortical areas, we performed correlation analysis of $h$ values measured between common 713 cortical source areas and paired visual targets (Figure 7h). We hypothesized that if the measured $714 h$ index faithfully reflects the hierarchical distance between the target area and the source area, the

715 difference between $h$ values measured for a common source area and paired target areas would be 716 the hierarchical distance between the two target areas. This relationship would be translated into a 717 best-fit line with a slope equal to 1 and an intercept indicating the hierarchical distance between 718 the two target areas when plotting paired $h$ values measured for common cortical source areas. We 719 compared the paired $h$ values between cortical source areas and five target visual areas, with 720 VISpm excluded for lack of L4 experiments and VISa excluded for low sample size. Overall, we

721 found a fair correspondence between the best fit lines based on the least-squares criterion and the 722 best fit with a slope equal to 1 . We then fit a linear regression model to the measured $h$ values 723 between cortical source areas and the five visual areas with the hierarchical level of VISp set at 724 zero. The relative hierarchical orders of cortical areas were estimated to best predict the measured $725 h$ index values. A strong correlation $\left(\mathrm{R}^{2}=0.94\right)$ was found between the predicted $h$ values and the 726 measured values (Figure 7i). The estimated hierarchical levels for the visual areas (Figure 7j) are

727 overall consistent with the predicted hierarchy based on the cell-type-specific projection ${ }^{49}$, with 728 the exception that the linear model fitting $h$-index values places VISa at the top of the dorsal stream 729 instead of VISam. Consistent with our previous findings, we find that the hierarchy in the mouse 730 visual cortex is shallow, especially for the dorsal stream.

732 Discussion

733 Here we present the construction and validation of a retrograde connectome pipeline for 734 the mouse brain, with a focus on the visual cortical areas. With improved virus tools and informatic 735 processing, our pipeline can be utilized to conduct large-scale systematic mapping of brain-wide 
presynaptic inputs at the cell class or type level. Together with our anterograde projection mapping pipeline, the current work proves the feasibility to build a comprehensive, directional, and 3D connectional atlas of the mouse brain at the cell type level.

\section{Target, layer and cell class together determine the presynaptic input patterns}

Our retrograde connectome dataset reveals that the presynaptic inputs to defined neuronal 742 classes are determined predominantly by the target area, followed by layer distribution of starter 743 cells and Cre line-defined cell classes. Both VISp and HVAs receive the most inputs from the 744 isocortex, followed by the thalamus and HPF. Strong cortical inputs are often from source areas 745 that receive strong visual area inputs, indicating reciprocal connections between the visual cortex 746 and other cortical modules. Each target area in the visual cortex exhibits unique input patterns, 747 distinguishing the dorsal stream targets from the ventral stream targets, and the anterolateral HVAs 748 from the anteromedial HVAs. In contrast to the highly differential cell-class-specific anterograde 749 projection patterns ${ }^{49}$, rabies tracings from Cre-defined cell classes in the same target reveal overall 750 similar global input patterns. We have obtained similar results when we applied the same approach 751 described in this study to another mouse cortical area, the primary motor cortex ${ }^{78}$. Nonetheless, 752 we also observe quantitative differences in inputs to layer- and Cre-defined starter cell classes. 753 Consistent with the feedforward thalamocortical connectivity from LGd to VISp, we find that L4 754 excitatory neurons receive the highest LGd input and lowest higher-order brain area inputs as 755 compared to excitatory neurons in other layers. We also discover that L6 neurons receive more 756 contralateral cortical inputs than any other layers, despite that the intracortical projections of L6 757 neurons are often locally restricted. Among the interneurons, we also find that inputs to 758 interneurons are mainly determined by target area and layer distribution of starter cells, although 759 cell-class-dependent local and long-range inputs can also be observed. Our observation is in line 760 with a recent rabies based tracing study which found strong similarity in local and long-range 761 inputs to L2/3 excitatory and inhibitory neuron types and significantly lower fraction of 762 contralateral inputs to these L2/3 neurons as compared to L6 Ntsr1 neurons ${ }^{79}$.

\section{Layer 4 neurons in visual cortex send significant inputs to other cortical areas}

Our study reveals that the role of L4 excitatory neurons is not limited to being the receiver 
interareal connections. In particular, VISp inputs to SSp-bfd-rll are almost exclusively from L4,

768 and similar preference for L4 inputs is observed for the connection between other HVAs and SSp-

769 bfd-rll. For HVA inputs to VISp, most of the input cells are in deep layers of HVAs, consistent

770 with the classical dogma of feedback information flow, while substantial amounts of inputs reside

771 in L4 of HVAs, suggesting a broad role of L4 neurons in the processing of visual information.

772 Being the major subclass of neurons receiving thalamic inputs while also sending outputs to HVAs,

773 VISp L4 neurons could in principle facilitate direct integrated processing of visual information.

774 Recent studies also found that L4 neurons send long distance projections and are the major source

775 of visual cortex projections to Pvalb neurons in barrel field ${ }^{40}$, consistent with a more complex role

776 of L4 excitatory neurons in interareal connectivity.

\section{Distinct patterns of thalamic projections to the visual areas}

The thalamus sends the second most abundant inputs to the visual areas. VISp is distinct

780 from the HVAs by preferentially receiving inputs from LGd as compared to LP, and L4 of the

781 VISp receives the most abundant LGd inputs as compared to other layers, consistent with the

782 feedforward flow of visual information from the LGd to L4 of the VISp. The overall patterns of 783 thalamic inputs to the HVAs show clear correlation with the anatomical proximity of HVAs. VISrl 784 and SSp-bfd-rll are either bordering or located in barrel cortex, and both receive abundant inputs 785 from the VPM, reminiscent of the strong VPM input to neurons in barrel cortex ${ }^{40,80}$. The SSp-bfd786 rll also receives substantial projections from anterior VISp but barely from posterior VISp ${ }^{49,50}$. In 787 this study, we find that SS-bfd-rll sends projections back to visual areas, demonstrating reciprocal 788 connections between them ${ }^{81}$. Unlike other HVAs that receive the highest proportion of thalamic 789 inputs from LP, SSp-bfd-rll distinctively receives the most inputs from PO and VPM, suggesting 790 that SSp-bfd-rll could be a transition area integrating the processing of both visual and 791 somatosensory information.

\section{Hierarchical organization of the mouse visual cortex}

794 We explore the possibility of predicting the hierarchical positions of mouse visual cortical 795 areas by the laminar distribution patterns of presynaptic neurons, an alternative approach from 796 predictions based on laminar termination patterns of axon projections ${ }^{49}$. The feedback and 797 feedforward connections in the mouse visual areas are gauged by the ratio of L2/3/4 inputs to 
L2/3/4/5 inputs. Using this quantitative parameter, we obtain a shallow hierarchy of the visual 799 areas, which places VISp at the bottom, and VISa and VISpor at the top of the hierarchy in the dorsal stream and the ventral stream, respectively. This hierarchy is largely consistent with the one we previously derived based on axon termination patterns ${ }^{49}$, and the anatomical hierarchy revealed by both output and input connectivity patterns mirrors the functional hierarchical organization of mouse cortical visual areas ${ }^{82}$. Our retrograde tracing shows VISa at the top of dorsal stream hierarchy, which is different from anterograde tracing showing VISam at the top. In primates, temporal association cortex (TE) in the ventral visual cortical pathway has large overlapping visual receptive field with no clearly separated visuotopic map ${ }^{66,83}$. Similarly, VISa in mouse dorsal stream has larger receptive field than VISam ${ }^{53}$, and VISa doesn't have a complete visual field, whereas VISam does ${ }^{54,55}$. Our current study also demonstrates that VISa receives more input from SSp than VISam does (Figure 3c). These differences between VISa and VISam support our current finding that VISa is higher in hierarchical level than VISam. Since we used cell-class specific Cre lines to define the starter cells in retrograde tracing, a comprehensive coverage of cell types in the target is a prerequisite for defining the hierarchical orders using presynaptic input patterns. Compared to our previous larger-scale anterograde projection study covering nearly the entire corticothalamic system ${ }^{49}$, our current study is restricted to the visual cortex and has varying levels of coverage for different visual areas. As we continue our effort to build the Allen Mouse Brain Connectivity Atlas, the brain-wide cellular-level retrograde connectome will enable a more in-

\section{Limitations of rabies virus tracing}

The monosynaptic rabies virus tracing system is a powerful tool in its ability to selectively infect starter cells and label only the first-order presynaptic neurons. However, although we have improved our virus tools to further enhance specificity and efficiency, there are still limitations of this strategy, which should be taken into consideration when interpreting the results.

Due to the high affinity between TVA and EnvA, low-level leaky expression of TVA in the absence of Cre is sufficient for rabies infection $29,32,34,61$. Although the leaky expression of RG

826 is often too low to allow trans-synaptic transportation of rabies virus, these cells can be mistakenly 827 counted as local trans-synaptically labeled cells. Our AAV helper virus is specifically designed to 828 reduce spurious expression in the absence of Cre by utilizing TVA ${ }^{66 \mathrm{~T}}$. However, many of the Cre- 
829 driver lines also have expression in areas sending input to the visual area, and AAV1 serotype is 830 known for its ability to retrogradely label the soma by traveling along the axon. It is possible that 831 the AAV helper virus can infect neurons in the brain area with direct input to the visual area, in 832 effect creating new starter cells, leading to the labeling of neurons in areas without direct 833 connection with the visual area. Therefore, independent connection mapping strategies are 834 required to verify novel connections revealed by rabies tracing.

835 On the other hand, it is also possible that the rabies virus tracing system does not reveal all 836 presynaptic neurons, even though we used the CVS-N2c strain which has higher trans-synaptic

837 efficiency. Rabies virus may not cross all synapses with equal efficiency, leading to preferential 838 representation of certain cell types within the presynaptic connectome. The efficiency of the trans839 synaptic spread of rabies virus is also affected by the expression level of RG in the starter neurons, 840 which is in turn limited by the expression of Cre from the driver lines, the titers of the AAV helper 841 virus and rabies virus tracer, as well as the amount of virus particles successfully delivered to the 842 target sites. The potential incompleteness and bias of the retrograde connectome mapped by the 843 rabies tracing system should always be considered, especially when an understanding of the 844 absolute number and the strength of synaptic connections between the input cells and the starter 845 cells is desired. 
848 H.Z., J.A.H., A.C., S.M. and S.Y. contributed to overall project design. A.C. designed and 849 orchestrated the viral tracing technology as well as viral production capability and established 850 these with the help from S.Y. T.Z. and M.T.M. S.Y., T.Z. and M.T.M. performed virus production. 851 A.C., S.Y., T.L.D. and B.O. conducted initial proof-of-principle studies. A.W. and P.A.G. 852 supervised surgical procedures with contributions from B.O., C.N., K.M., S.L, A.C., L.C., K.N., 853 N.H., E.G., J.L., R.A, R.H., and J.S. P.A.G. supervised ISI procedures with contributions from 854 S.C., S.S., E.K.L., F.G., and T.N. M. McGraw. supervised histological processing with 855 contributions from T.E., J.B., M. Maxwell., H.G., A.G., K.B., and A.R. P.R.N. coordinated 856 imaging procedures with contributions from R.E., M.G., S.R., L.P., N.I.D., N.K.N., and M.J.T. 857 L.N., L.K. and W.W. performed informatics data processing. K.E.H. and S.Y. coordinated 858 workflow and carried out quality control. M.N. contributed to the development of data 859 visualization tools. Q.W., S.M., J.A.H., A.C., S.Y. and H.Z. formulated data generation and 860 analysis strategies. S.Y. analyzed data and prepared figures. S.Y., B.T., and H.Z. wrote the manuscript with inputs from all authors.

\section{Declaration of Interests}

864 J.A.H., K.N., K.E.H. and P.R.N. are currently employed by Cajal Neuroscience.

\section{Acknowledgment}

We are grateful to the Transgenic Colony Management, Neurosurgery \& Behavior, Lab Animal Services, Molecular Genetics, Imaging, Histology, Technology, and Project Management teams at the Allen Institute for technical and management support. We thank Thomas R Reardon, Andrew J Murray and Ian Wickersham for providing cell lines and plasmids for the establishment of rabies virus production at the Allen Institute. This work was supported by the Allen Institute for

872 Brain Science and by the National Institute of Mental Health (NIMH) of the National Institutes of 873 Health (NIH) under award number U19MH114830 to H.Z. The content is solely the responsibility 874 of the authors and does not necessarily represent the official views of NIH and its subsidiary 875 institutes. We thank the Allen Institute founder, Paul G. Allen, for his vision, encouragement, and 876 support. 
8801 White, J. G., Southgate, E., Thomson, J. N. \& Brenner, S. The structure of the nervous $881 \quad$ system of the nematode Caenorhabditis elegans. Philos Trans R Soc Lond B Biol Sci 314, $882 \quad 1-340$, doi:10.1098/rstb.1986.0056 (1986).

8832 Varshney, L. R., Chen, B. L., Paniagua, E., Hall, D. H. \& Chklovskii, D. B. Structural 884 properties of the Caenorhabditis elegans neuronal network. PLoS Comput Biol 7, e1001066, doi:10.1371/journal.pcbi.1001066 (2011).

887 437-444, doi:10.1126/science.1221762 (2012).

8884 Hildebrand, D. G. C. et al. Whole-brain serial-section electron microscopy in larval zebrafish. Nature 545, 345-349, doi:10.1038/nature22356 (2017).

891

$892 \quad 6$

893

894

895

$896 \quad 8$

897 Zheng, Z. et al. A Complete Electron Microscopy Volume of the Brain of Adult Drosophila melanogaster. Cell 174, 730-743 e722, doi:10.1016/j.cell.2018.06.019 (2018).

6 Scheffer, L. K. et al. A connectome and analysis of the adult Drosophila central brain. Elife 9, doi:10.7554/eLife.57443 (2020).

7 Witvliet, D. et al. Connectomes across development reveal principles of brain maturation. Nature 596, 257-261, doi:10.1038/s41586-021-03778-8 (2021).

8 Joesch, M. et al. Reconstruction of genetically identified neurons imaged by serial-section electron microscopy. Elife 5, doi:10.7554/eLife.15015 (2016).

9 Yin, W. et al. A petascale automated imaging pipeline for mapping neuronal circuits with high-throughput transmission electron microscopy. Nat Commun 11, 4949, doi:10.1038/s41467-020-18659-3 (2020).

904

905 Lee, W. C. et al. Anatomy and function of an excitatory network in the visual cortex. Nature 532, 370-374, doi:10.1038/nature17192 (2016).

11 Morgan, J. L., Berger, D. R., Wetzel, A. W. \& Lichtman, J. W. The Fuzzy Logic of Network Connectivity in Mouse Visual Thalamus. Cell 165, 192-206, doi:10.1016/j.cell.2016.02.033 (2016).

12 Kasthuri, N. et al. Saturated Reconstruction of a Volume of Neocortex. Cell 162, 648-661, doi:10.1016/j.cell.2015.06.054 (2015).

13 Gong, H. et al. High-throughput dual-colour precision imaging for brain-wide connectome with cytoarchitectonic landmarks at the cellular level. Nat Commun 7, 12142, doi:10.1038/ncomms12142 (2016).

14 Winnubst, J. et al. Reconstruction of 1,000 Projection Neurons Reveals New Cell Types and Organization of Long-Range Connectivity in the Mouse Brain. Cell 179, 268-281 e213, doi:10.1016/j.cell.2019.07.042 (2019). Jiang, X. et al. Principles of connectivity among morphologically defined cell types in adult neocortex. Science 350, aac9462, doi:10.1126/science.aac9462 (2015). neocortical excitatory connections. Nature 457, 1142-1145, doi:10.1038/nature07709 (2009).

1 Petreanu, L., Huber, D., Sobczyk, A. \& Svoboda, K. Channelrhodopsin-2-assisted circuit mapping of long-range callosal projections. Nat Neurosci 10, 663-668, doi:10.1038/nn1891 (2007). 
92218 Avermann, M., Tomm, C., Mateo, C., Gerstner, W. \& Petersen, C. C. Microcircuits of excitatory and inhibitory neurons in layer $2 / 3$ of mouse barrel cortex. J Neurophysiol 107, 3116-3134, doi:10.1152/jn.00917.2011 (2012).

19 Izquierdo-Serra, M., Hirtz, J. J., Shababo, B. \& Yuste, R. Two-Photon Optogenetic Mapping of Excitatory Synaptic Connectivity and Strength. iScience 8, 15-28, doi:10.1016/j.isci.2018.09.008 (2018).

20 Mao, T. et al. Long-range neuronal circuits underlying the interaction between sensory and motor cortex. Neuron 72, 111-123, doi:10.1016/j.neuron.2011.07.029 (2011).

21 Mohammed, A. I. et al. An integrative approach for analyzing hundreds of neurons in task doi:10.1038/srep20986 (2016).

22 Callaway, E. M. Transneuronal circuit tracing with neurotropic viruses. Curr Opin Neurobiol 18, 617-623, doi:10.1016/j.conb.2009.03.007 (2008).

23 Callaway, E. M. \& Luo, L. Monosynaptic Circuit Tracing with Glycoprotein-Deleted Rabies Viruses. J Neurosci 35, 8979-8985, doi:10.1523/JNEUROSCI.0409-15.2015 (2015).

24 Beier, K. T. et al. Rabies screen reveals GPe control of cocaine-triggered plasticity. Nature 549, 345-350, doi:10.1038/nature23888 (2017).

25 Kim, E. J., Jacobs, M. W., Ito-Cole, T. \& Callaway, E. M. Improved Monosynaptic Neural Circuit Tracing Using Engineered Rabies Virus Glycoproteins. Cell Rep 15, 692-699, doi:10.1016/j.celrep.2016.03.067 (2016).

26 Lo, L. \& Anderson, D. J. A Cre-dependent, anterograde transsynaptic viral tracer for mapping output pathways of genetically marked neurons. Neuron 72, 938-950, doi:10.1016/j.neuron.2011.12.002 (2011).

27 Lo, L. et al. Connectional architecture of a mouse hypothalamic circuit node controlling social behavior. Proc Natl Acad Sci U S A 116, 7503-7512, doi:10.1073/pnas.1817503116 (2019).

28 Miyamichi, K. et al. Cortical representations of olfactory input by trans-synaptic tracing. Nature 472, 191-196, doi:10.1038/nature09714 (2011).

29 Miyamichi, K. et al. Dissecting local circuits: parvalbumin interneurons underlie broad feedback control of olfactory bulb output. Neuron 80, 1232-1245, doi:10.1016/j.neuron.2013.08.027 (2013).

30 Osakada, F. et al. New rabies virus variants for monitoring and manipulating activity and gene expression in defined neural circuits. Neuron 71, 617-631, doi:10.1016/j.neuron.2011.07.005 (2011).

31 Wall, N. R., Wickersham, I. R., Cetin, A., De La Parra, M. \& Callaway, E. M. Monosynaptic circuit tracing in vivo through Cre-dependent targeting and complementation of modified rabies virus. Proc Natl Acad Sci U S A 107, 21848-21853, doi:10.1073/pnas.1011756107 (2010).

32 Watabe-Uchida, M., Zhu, L., Ogawa, S. K., Vamanrao, A. \& Uchida, N. Whole-brain mapping of direct inputs to midbrain dopamine neurons. Neuron 74, 858-873, doi:10.1016/j.neuron.2012.03.017 (2012).

33 Weible, A. P. et al. Transgenic targeting of recombinant rabies virus reveals monosynaptic connectivity of specific neurons. $J$ Neurosci 30, 16509-16513, doi:10.1523/JNEUROSCI.2442-10.2010 (2010). 
96734 Weissbourd, B. et al. Presynaptic partners of dorsal raphe serotonergic and GABAergic 968 neurons. Neuron 83, 645-662, doi:10.1016/j.neuron.2014.06.024 (2014).

$96935 \quad$ Wickersham, I. R., Finke, S., Conzelmann, K. K. \& Callaway, E. M. Retrograde neuronal tracing with a deletion-mutant rabies virus. Nat Methods 4, 47-49, doi:10.1038/nmeth999 (2007).

36 Wickersham, I. R. et al. Monosynaptic restriction of transsynaptic tracing from single, genetically targeted neurons. Neuron 53, 639-647, doi:10.1016/j.neuron.2007.01.033 (2007).

975

37 Reardon, T. R. et al. Rabies Virus CVS-N2c(DeltaG) Strain Enhances Retrograde Synaptic Transfer and Neuronal Viability. Neuron 89, 711-724, doi:10.1016/j.neuron.2016.01.004 (2016).

Chatterjee, S. et al. Nontoxic, double-deletion-mutant rabies viral vectors for retrograde targeting of projection neurons. Nat Neurosci 21, 638-646, doi:10.1038/s41593-018-00917 (2018).

39 Ciabatti, E., Gonzalez-Rueda, A., Mariotti, L., Morgese, F. \& Tripodi, M. Life-Long Genetic and Functional Access to Neural Circuits Using Self-Inactivating Rabies Virus. Cell 170, 382-392 e314, doi:10.1016/j.cell.2017.06.014 (2017).

40 Hafner, G. et al. Mapping Brain-Wide Afferent Inputs of Parvalbumin-Expressing GABAergic Neurons in Barrel Cortex Reveals Local and Long-Range Circuit Motifs. Cell Rep 28, 3450-3461 e3458, doi:10.1016/j.celrep.2019.08.064 (2019).

41 Madisen, L. et al. A robust and high-throughput Cre reporting and characterization system for the whole mouse brain. Nat Neurosci 13, 133-140, doi:10.1038/nn.2467 (2010).

42 Daigle, T. L. et al. A Suite of Transgenic Driver and Reporter Mouse Lines with Enhanced Brain-Cell-Type Targeting and Functionality. Cell 174, 465-480 e422, doi:10.1016/j.cell.2018.06.035 (2018).

43 Tasic, B. et al. Adult mouse cortical cell taxonomy revealed by single cell transcriptomics. Nat Neurosci 19, 335-346, doi:10.1038/nn.4216 (2016).

44 Tasic, B. et al. Shared and distinct transcriptomic cell types across neocortical areas. Nature 563, 72-78, doi:10.1038/s41586-018-0654-5 (2018).

45 Fenno, L. E. et al. Targeting cells with single vectors using multiple-feature Boolean logic. Nat Methods 11, 763-772, doi:10.1038/nmeth.2996 (2014).

46 Atasoy, D., Aponte, Y., Su, H. H. \& Sternson, S. M. A FLEX switch targets Channelrhodopsin-2 to multiple cell types for imaging and long-range circuit mapping. $J$ Neurosci 28, 7025-7030, doi:10.1523/JNEUROSCI.1954-08.2008 (2008).

47 Harris, J. A. et al. Anatomical characterization of Cre driver mice for neural circuit mapping and manipulation. Front Neural Circuits 8, 76, doi:10.3389/fncir.2014.00076 (2014). tracing with fluorescent proteins in nontransgenic and cre driver mice. Curr Protoc Neurosci Chapter 1, Unit 120 21-18, doi:10.1002/0471142301.ns0120s59 (2012).

1008 Harris, J. A. et al. Hierarchical organization of cortical and thalamic connectivity. Nature 575, 195-202, doi:10.1038/s41586-019-1716-z (2019).

1010 doi:10.1038/nature13186 (2014).

1012 Wang, Q. et al. The Allen Mouse Brain Common Coordinate Framework: A 3D Reference Atlas. Cell 181, 936-953 e920, doi:10.1016/j.cell.2020.04.007 (2020). 
101352 Kuan, L. et al. Neuroinformatics of the Allen Mouse Brain Connectivity Atlas. Methods

1014

1015

1016

1017

1018

1019

1020

1021

1022

1023

1024

1025

1026

1027

1028

1029

1030

1031

1032

1033

1034

1035

1036

1037

1038

1039

1040

1041

1042

1043

1044

1045

1046

1047

1048

1049

1050

1051

1052

1053

1054

1055

1056 73, 4-17, doi:10.1016/j.ymeth.2014.12.013 (2015).

53 Wang, Q. \& Burkhalter, A. Area map of mouse visual cortex. J Comp Neurol 502, 339357, doi:10.1002/cne.21286 (2007).

54 Zhuang, J. et al. An extended retinotopic map of mouse cortex. Elife 6, doi:10.7554/eLife.18372 (2017).

55 Garrett, M. E., Nauhaus, I., Marshel, J. H. \& Callaway, E. M. Topography and areal organization of mouse visual cortex. $J$ Neurosci 34, 12587-12600, doi:10.1523/JNEUROSCI.1124-14.2014 (2014).

56 Gamanut, R. et al. The Mouse Cortical Connectome, Characterized by an Ultra-Dense Cortical Graph, Maintains Specificity by Distinct Connectivity Profiles. Neuron 97, 698715 e610, doi:10.1016/j.neuron.2017.12.037 (2018).

57 Wang, Q., Sporns, O. \& Burkhalter, A. Network analysis of corticocortical connections reveals ventral and dorsal processing streams in mouse visual cortex. J Neurosci 32, 43864399, doi:10.1523/JNEUROSCI.6063-11.2012 (2012).

58 Felleman, D. J. \& Van Essen, D. C. Distributed hierarchical processing in the primate cerebral cortex. Cereb Cortex 1, 1-47, doi:10.1093/cercor/1.1.1 (1991).

59 Markov, N. T. et al. Anatomy of hierarchy: feedforward and feedback pathways in macaque visual cortex. J Comp Neurol 522, 225-259, doi:10.1002/cne.23458 (2014).

60 Kohara, K. et al. Cell type-specific genetic and optogenetic tools reveal hippocampal CA2 circuits. Nat Neurosci 17, 269-279, doi:10.1038/nn.3614 (2014).

61 Do, J. P. et al. Cell type-specific long-range connections of basal forebrain circuit. Elife 5, doi:10.7554/eLife.13214 (2016).

62 Zawadzka, M. et al. CNS-resident glial progenitor/stem cells produce Schwann cells as well as oligodendrocytes during repair of CNS demyelination. Cell Stem Cell 6, 578-590, doi:10.1016/j.stem.2010.04.002 (2010).

63 Koni, P. A. et al. Conditional vascular cell adhesion molecule 1 deletion in mice: impaired lymphocyte migration to bone marrow. $J$ Exp Med 193, 741-754, doi:10.1084/jem.193.6.741 (2001).

64 Octeau, J. C. et al. An Optical Neuron-Astrocyte Proximity Assay at Synaptic Distance Scales. Neuron 98, 49-66 e49, doi:10.1016/j.neuron.2018.03.003 (2018).

65 Kravitz, D. J., Saleem, K. S., Baker, C. I. \& Mishkin, M. A new neural framework for visuospatial processing. Nat Rev Neurosci 12, 217-230, doi:10.1038/nrn3008 (2011).

66 Kravitz, D. J., Saleem, K. S., Baker, C. I., Ungerleider, L. G. \& Mishkin, M. The ventral visual pathway: an expanded neural framework for the processing of object quality. Trends Cogn Sci 17, 26-49, doi:10.1016/j.tics.2012.10.011 (2013).

67 Cenquizca, L. A. \& Swanson, L. W. Spatial organization of direct hippocampal field CA1 axonal projections to the rest of the cerebral cortex. Brain Res Rev 56, 1-26, doi:10.1016/j.brainresrev.2007.05.002 (2007).

68 Wang, Q. et al. Organization of the connections between claustrum and cortex in the mouse. J Comp Neurol 525, 1317-1346, doi:10.1002/cne.24047 (2017).

69 Huppe-Gourgues, F., Jegouic, K. \& Vaucher, E. Topographic Organization of Cholinergic Innervation From the Basal Forebrain to the Visual Cortex in the Rat. Front Neural Circuits 12, 19, doi:10.3389/fncir.2018.00019 (2018). 
105770 Li, X. et al. Generation of a whole-brain atlas for the cholinergic system and mesoscopic 1058 projectome analysis of basal forebrain cholinergic neurons. Proc Natl Acad Sci U S A 115, 1059 415-420, doi:10.1073/pnas.1703601115 (2018).

$106071 \quad$ Bari, B. A., Chokshi, V. \& Schmidt, K. Locus coeruleus-norepinephrine: basic functions $1061 \quad$ and insights into Parkinson's disease. Neural Regen Res 15, 1006-1013, doi:10.4103/1673$1062 \quad 5374.270297$ (2020).

106372 Schwarz, L. A. et al. Viral-genetic tracing of the input-output organization of a central 1064 noradrenaline circuit. Nature 524, 88-92, doi:10.1038/nature14600 (2015).

1066 Ren, J. et al. Single-cell transcriptomes and whole-brain projections of serotonin neurons in the mouse dorsal and median raphe nuclei. Elife 8, doi:10.7554/eLife.49424 (2019).

1068

1069

1070

1071 Goodale, M. A. \& Milner, A. D. Separate visual pathways for perception and action. Trends Neurosci 15, 20-25, doi:10.1016/0166-2236(92)90344-8 (1992).

75 Antonini, A., Fagiolini, M. \& Stryker, M. P. Anatomical correlates of functional plasticity in mouse visual cortex. J Neurosci 19, 4388-4406 (1999).

1072

76 Humphrey, A. L., Sur, M., Uhlrich, D. J. \& Sherman, S. M. Projection patterns of

1073

1074 individual $\mathrm{X}$ - and $\mathrm{Y}$-cell axons from the lateral geniculate nucleus to cortical area 17 in the cat. J Comp Neurol 233, 159-189, doi:10.1002/cne.902330203 (1985).

1075

1076

1077

1078

1079

1080

1081

1082

1083

1084

1085

1086

1087

1088

1089

1090

77 LeVay, S. \& Gilbert, C. D. Laminar patterns of geniculocortical projection in the cat. Brain Res 113, 1-19, doi:10.1016/0006-8993(76)90002-0 (1976).

78 Muñoz-Castañeda, R. et al. Cellular Anatomy of the Mouse Primary Motor Cortex. bioRxiv, 2020.2010.2002.323154, doi:10.1101/2020.10.02.323154 (2020).

79 Brown, A. P. Y. et al. Analysis of segmentation ontology reveals the similarities and differences in connectivity onto L2/3 neurons in mouse V1. Sci Rep 11, 4983, doi:10.1038/s41598-021-82353-7 (2021).

80 DeNardo, L. A., Berns, D. S., DeLoach, K. \& Luo, L. Connectivity of mouse somatosensory and prefrontal cortex examined with trans-synaptic tracing. Nat Neurosci 18, 1687-1697, doi:10.1038/nn.4131 (2015).

81 Masse, I. O., Ross, S., Bronchti, G. \& Boire, D. Asymmetric Direct Reciprocal Connections Between Primary Visual and Somatosensory Cortices of the Mouse. Cereb Cortex 27, 4361-4378, doi:10.1093/cercor/bhw239 (2017).

82 Siegle, J. H. et al. Survey of spiking in the mouse visual system reveals functional hierarchy. Nature 592, 86-92, doi:10.1038/s41586-020-03171-x (2021).

83 Gattass, R. et al. Cortical visual areas in monkeys: location, topography, connections, columns, plasticity and cortical dynamics. Philos Trans R Soc Lond B Biol Sci 360, 709731, doi:10.1098/rstb.2005.1629 (2005).

1092 


\section{METHODS}

All experimental procedures related to the use of mice were approved by the Institutional Animal Care and Use Committee of the Allen Institute for Brain Science, in accordance with NIH 1096 guidelines.

\section{Outline of the data generation and processing pipeline}

A standardized data generation and processing platform was established. Mice first 1100 received virus injection, and the brains were then imaged by serial two-photon tomography (STPT). 1101 Images passed annotation quality control (QC) were subject to data processing through the 1102 informatics pipeline, while brain sections around the injection sites were mounted, immunostained 1103 to enhance the red fluorophore and imaged by confocal microscopy to identify starter cells. 1104 Specimens failed the staining QC and starter cell QC were removed from the pipeline, and finally, 1105 artifacts in informatics processing were identified and corrected through segmentation QC.

\section{Data Availability}

1108 Plasmids for the generation of recombinant viruses will be deposited in Addgene. All 1109 anterograde tracing data (including high resolution STPT images, and informatically processed 1110 axonal projection across brain structures) are available through the Allen Mouse Brain 1111 Connectivity Atlas portal (http://connectivity.brain-map.org/). A link for each anterograde tracing 1112 experiment is provided in Supplementary Tables 4 and 5. Original images for transsynaptic rabies 1113 viral tracing will be available through the Brain Image Library 1114 (https://www.brainimagelibrary.org/). Normalized presynaptic input volumes across the brain for 1115 all rabies virus tracing experiments are listed in Supplementary Table 3. Original retrograde 1116 labeling data from initial informatic quantification are available upon request.

\section{Animals}

1119 To identify presynaptic inputs to different neuronal populations in the visual cortex, 14 1120 Cre-transgenic mice with distinct cell type and layer labeling patterns (aged 2-6 months, either 1121 gender depending on availability) were used. These Cre lines have been previously utilized 1122 together with anterograde AAV viral tracers for the construction of the Allen Mouse Brain 1123 Connectivity Atlas. The expression patterns of these lines can be found in the Allen Institute 
1124 Transgenic Characterization data portal (http://connectivity.brain-map.org/transgenic). To 1125 quantify the spurious rabies virus labeling, wild-type animals were used, and to compare different 1126 recombinant rabies and AAV helper viruses, Cre transgenic mice labeling neurons and non1127 neuronal cells were used. The specific genotypes used for each experiment are listed in 1128 Supplementary Tables 1 and 2. Mice were housed under 14h:10h light-dark cycle with ad libitum 1129 access to food and water.

\section{Virus design, preparation and titer information}

$A A V$ viruses and rabies viruses used in the mesoscale retrograde connectome pipeline were 1133 generated in the Allen Institute of Brain Science. Our AAV helper viruses utilize the FELX 1134 strategy and contain the tricistronic cassettes of Syn-DIO-TVA-dTomato-RV G, followed by a 1135 short bovine growth hormone polyadenylation sequence. The Kozak sequences and the starter 1136 codon of TVA are located 5' to the FLEX switch, while the TVA (lacking a start codon)-P2A1137 dTom-P2A-RV G cassette is within the FLEX cassette and inverted respective to the promoter. 1138 The AAV helper viruses selected for the mesoscale retrograde connectome pipeline utilized a 1139 mutant TVA, TVA ${ }^{66 \mathrm{~T}}$, and the RV G from the CVS N2c strain.

1140 The AAV1 serotype of the helper virus was produced using a helper-free HEK293 cell 1141 system followed by iodixanol gradient purification. Multiple batches of AAV1-Syn-DIO-TVA ${ }^{66}$ -

1142 dTom-CVS N2cG viruses were used in the course of the mesoscale connectivity project, and the 1143 titers of the viruses were in the range of $2 \times 10^{12}$ to $1 \times 10^{13} \mathrm{GC} / \mathrm{ml}$.

1144 The CVS N2 $\mathrm{c}^{\mathrm{dG}}$-H2B-EGFP rabies virus was generated by replacing GFP in the rabies 1145 genomic plasmid RabV CVS-N2c(deltaG)-EGFP (Addgene, Plasmid \#73461) with H2B-EGFP 1146 flanked by 5' XmaI and 3' NheI-KasI sites. EnvA CVS N2 ${ }^{\mathrm{dG}}$-H2B-EGFP rabies viruses were 1147 generated from the genomic plasmid as described previously ${ }^{1}$. The titer of EnvA CVS N2 $\mathrm{c}^{\mathrm{dG}}-$ 1148 H2B-EGFP rabies virus used in the study was adjusted to be around $5 \times 10^{9} \mathrm{GC} / \mathrm{ml}$.

1149 The SAD B19 $9^{\mathrm{dG}}-\mathrm{H} 2 \mathrm{~B}-E G F P$ virus was generated by inserting the H2B-EGFP sequence 1150 into the gG locus of the pSADdeltaG-F3 plasmid (Addgene, Plasmid \#32634). The EnvA SAD $1151 \mathrm{~B} 19^{\mathrm{dG}}-\mathrm{H} 2 \mathrm{~B}-\mathrm{EGFP}$ rabies viruses were generated from the genomic plasmid as described 1152 previously ${ }^{2}$. The EnvA SAD B19 ${ }^{\mathrm{dG}}-\mathrm{GFP}$ was from Salk Institute. Both EnvA SAD B19 $9^{\mathrm{dG}}-\mathrm{H}_{2} \mathrm{~B}-$ 1153 EGFP and EnvA SAD B19 ${ }^{\mathrm{dG}}-\mathrm{GFP}$ viruses were diluted to $5 \times 10^{9} \mathrm{GC} / \mathrm{ml}$ to match that of the EnvA 1154 CVS N2 $\mathrm{c}^{\mathrm{dG}}-\mathrm{H} 2 \mathrm{~B}-\mathrm{EGFP}$ virus. 


\section{Surgery}

All mice received unilateral injection into a single target region in the left hemisphere. For 1158 monosynaptic retrograde tracing of whole brain inputs to Cre-defined cell populations, the AAV 1159 helper virus was injected first into the target site, followed $21 \pm 3$ days later by another injection in 1160 the same location with the EnvA CVS N2c ${ }^{\mathrm{dG}}-\mathrm{H} 2 \mathrm{~B}-\mathrm{EGFP}$ rabies virus. After one week survival, 1161 animals were sacrificed, and perfused with 4\% paraformaldehyde (PFA). Brains were dissected 1162 and post-fixed in 4\% PFA at room temperature for 3-6 $\mathrm{h}$ and then overnight at $4{ }^{\circ} \mathrm{C}$.

1163 To precisely target each visual area, functional mapping of visual field space by intrinsic 1164 signal imaging (ISI) was used to guide injection placement ${ }^{3}$. An image of the surface vasculature 1165 was acquired to provide fiduciary marker references on the surface of the brain. An overlay of the 1166 visual field map over the vasculature fiducials was used to identify the target injection site. For 1167 injections that failed repeatedly under the guidance of ISI, transcranial injections were conducted 1168 using stereotaxic injection coordinates specific for each target site. The anterior/posterior (AP) 1169 coordinates are referenced from the transverse sinus (TS), the medial/lateral (ML) coordinates are 1170 distance from midline at Bregma, and the dorsal/ventral (DV) coordinates are measured from the 1171 pial surface of the brain. Stereotaxic coordinates for each area are as follows: VISp (6 subareas) 1172 (VISp-1: AP:1.50(TS), ML:-2.55, and DV:0.3, 0.6; VISp-2: AP:2.59(TS), ML:-2.55, and DV:0.3, 1173 0.6; VISp-3: AP:1.90(TS), ML:-3.10, and DV:0.3, 0.6; VISp-4: AP:1.05(TS), ML:-3.50, and 1174 DV:0.3, 0.6; VISp-5: AP:0.75(TS), ML:-3.00, and DV:0.3, 0.6; VISp-6: AP:0.61(TS), ML:-2.10, 1175 and DV:0.3, 0.6), VISl (AP:1.4(TS), ML: -4.10, DV:0.3, 0.6); VISpm (AP:1.9(TS), ML: -1.60; 1176 DV:0.3, 0.6), VISam (AP: 3.0(TS), ML: -1.70, DV:0.3, 0.6), VISal (AP: 2.4(TS), ML: -3.70, 1177 DV:0.3, 0.6, Angle:15²), and VISrl (AP: 2.8(TS), ML:-3.30, DV: 0.3, 0.6). For some target areas, 1178 injections were made at two depths to label neurons throughout all six cortical layers. The AAV1 1179 helper virus was injected using the iontophoresis method, with current settings of $3 \mu \mathrm{A}, 7 \mathrm{sec}$ 1180 on/off cycles and $5 \mathrm{~min}$ total. The EnvA rabies viruses were injected using a nanoinjector, and a 1181 total of $500 \mathrm{nl}$ was delivered in $23 \mathrm{nl}$ increments over a $3 \mathrm{~min}$ and $10 \mathrm{sec}$ interval.

1182 Tamoxifen-inducible Cre line (CreER) mice were treated with $0.2 \mathrm{mg} / \mathrm{g}$ body weight of 1183 tamoxifen solution in corn oil via oral gavage once per day for 5 consecutive days starting the 1184 week following virus injection. Trimethoprim-inducible Cre line mice were treated with $0.3 \mathrm{mg} / \mathrm{g}$ 1185 body weight of trimethoprim solution in 10\% DMSO via oral gavage once per day for 3 
1186 consecutive days starting the week following AAV virus injection. Rabies viruses were injected 3

1187 weeks post induction. All mice were deeply anesthetized before intracardial perfusion, brain 1188 dissection, and tissue preparation for serial imaging.

\section{STPT}

The injected brains were imaged by STPT (TissueCyte 1000, TissueVision Inc. Somerville, 1192 MA) as described previously with a few modifications ${ }^{3,4}$. In brief, brains were embedded in 1193 agarose block, and imaged from the caudal end along the rostrocaudal z-axis. The specimen was 1194 illuminated with $925 \mathrm{~nm}$ wavelength light. Two-photon image tiles for red and green channels 1195 with a nominal resolution of $0.875 \mu \mathrm{m} \times 0.875 \mu \mathrm{m} \times 2 \mu \mathrm{m} \times-y-z$ were taken at $40 \mu \mathrm{m}$ below the 1196 cutting surface. The laser power and photo-multiplier tube (PMT) voltage were set at $190 \mathrm{~mW}$ 1197 (measured at the objective) and $\sim 600 \mathrm{~V}$ (equal on all channels). In order to compensate for

1198 variation between imaging systems and specimens, these parameters are adjusted on each imaging 1199 run using an observed level of autofluorescence in the red channel. The following procedures were 1200 conducted: locate the central canal of the brain stem; locate the surface of the tissue; adjust the 1201 objective piezo stage such that the image plane is $40 \mu \mathrm{m}$ deep in the specimen; move $700 \mu \mathrm{m}$ 1202 laterally, exposing an area of uniform tissue structure; adjust the PMT voltage such that the mean 1203 intensity of this area falls within the range of 600-650; from the central canal, the specimen is then 1204 centered laterally within the imaging area and the acquisition is commenced. After an entire brain 1205 section was imaged, a 100- $\mu$ m section was removed from the specimen by the vibratome, followed 1206 by imaging of the next plane. Scanned image tiles were stitched to form a single high-resolution 1207 image. Images from 140 sections were collected to cover the full range of mouse brain. Upon 1208 completion of imaging, sections were retrieved and stored in PBS with $0.1 \%$ sodium azide at $4^{\circ} \mathrm{C}$.

\section{Starter cell identification and quantification}

1211 The starter cells are those with both AAV helper virus and EnvA RV-H2B-EGFP infection, 1212 and thus have red fluorescence in the soma and green fluorescence in the nuclei. For starter cell 1213 quantification, TissueCyte brain sections were sorted according to the rostrocaudal axis. Around $121420100-\mu \mathrm{m}$ sections flanking the virus injection sites were identified, mounted on gelatin coated 1215 glass slides, and immunostained to enhance red fluorescence signal. The immunofluorescence 1216 staining was conducted using an automated slide stainer (Biocare, IntelliPATH FLX). Slides were 
1217 blocked in Image iT FX Signal Enhancer (Thermo Fisher Scientific Cat\# I3693) for 45 minutes, 1218 followed by 1-hour incubation in a blocking solution containing 1\% normal goat serum (Vector 1219 Laboratories Cat\#S1000) and 1\% Triton X (VWR). Sections were then incubated in the primary 1220 antibody solution (1\% goat serum, 1\% Triton X, Rockland Cat\# 600-401-379, RRID:AB_2209751, 1221 1:2000) for 1.5 hours, and then the in secondary antibody solution (1\% goat serum, $1 \%$ Triton $X$, 1222 and 1:500 goat anti-rabbit conjugated with Alexa Fluor 594, Thermo Fisher Scientific Cat\# A1223 11037, RRID:AB_2534095) for 2 hours at room temperature after rinsing with $0.1 \%$ Triton $\mathrm{X}$ 1224 wash solution. All sections were stained with $5 \mu \mathrm{M}$ Dapi (Thermo Fisher Scientific D1306) and 1225 coverslipped using Fluoromount G (Southern Biotech Cat\# 0100-01B). Stained sections were 1226 imaged using a Leica SP8 TCS confocal microscope under a 10x objective. Starter cells were 1227 counted in ImageJ using the Cell Counter plugin.

1228

\section{Image data QC and annotation}

The acquired TissueCyte images and the confocal images went through several steps of 1231 quality control processes: annotation QC, staining QC, starter cell QC, and segmentation QC.

1232 Specimens that did not pass any one of the QC steps were considered fails and removed from the 1233 pipeline. After TissueCyte imaging, specimens are assessed for surgical and imaging quality 1234 through Annotation QC. Failures at this step include no green signal, TissueCyte imaging error, 1235 tissue damages, and poor surgical targeting. Polygons are drawn around the injection site to link 1236 the injection site to the Allen Mouse Brain Common Coordinate Framework, version 3 (CCFv3). 1237 Specimens passed Annotation QC were sent for the next step of mounting, and immunostaining 1238 for starter cell identification. Staining QC identified and removed specimens in which no red1239 fluorescent cells were found after immunostaining-mediated enhancement of red fluorescence 1240 signal from the AAV helper virus. Starter cell QC further removed specimens in which no starter 1241 cells were identified after confocal imaging. Finally, specimens with errors in the subsequent 1242 informatics data pipeline steps were identified in the Segmentation QC step.

\section{Image data processing}

1245 Images were processed and registered to the CCFv3 through our informatics data pipeline 1246 (IDP $)^{5,6}$. The signal detection algorithm was modified to detect nuclear objects with high 1247 sensitivity, which accepts out of focus nuclei and has lower contrast requirements. In addition, 
1248 high intensity pixels near the detected objects were included into the signal pixel set. Detected 1249 objects near hyper-intense artifacts occurring in multiple channels were removed. The output is a 1250 full resolution mask that classifies each pixel as either signal or background. An isotropic 3D 1251 summary of each brain is constructed by dividing each image series into $10 \mu \mathrm{m} \times 10 \mu \mathrm{m} \times 10 \mu \mathrm{m}$ 1252 grid voxels. Total signal is computed for each voxel by summing the number of signal-positive 1253 pixels in that voxel. Each image stack is registered in a multi-step process using both global affine 1254 and local deformable registration to the 3D Allen mouse brain reference atlas as previously 1255 described ${ }^{5,6}$.

1257 Analysis of whole brain presynaptic inputs to the visual areas

1258 The accuracy of targeting was verified by overlaying the injection site polygon of each 1259 experiment to the ISI image or by identifying the anatomical structure where the injection centroid 1260 was located in the CCFv3. Since the signal detection algorithm was optimized to detect sparse 1261 presynaptic labeling with high sensitivity, the automatically detected volume of input signal can 1262 have false positives where high background signal is falsely identified as input signal. False 1263 positives tend to occur more frequently in brain structures with low input signal and high 1264 background fluorescence such as the cerebellum, and are rarely found in areas with strong input 1265 signals such as the isocortex. In order to remove this type of artifacts, we identified a set of 92 1266 negative brains that were processed through the pipeline, but showed no rabies-mediated GFP 1267 expression, and used this negative dataset to calculate the threshold of false positive signal, i.e., 1268 the value of mean input signal volume plus 6 standard deviations for each of the 314 ipsilateral 1269 and 314 contralateral major structures of the brain. Any structure not passing this threshold was 1270 set to "0". A manually validated binary mask was then applied to further remove artifacts in 1271 informatically-derived measures. Following these two steps, input signal volume in a given 1272 structure was normalized to the total input of the brain. The post-threshold, masked, normalized 1273 input signal volumes were used to build the weighted connectivity matrix.

1274 When analyzing the fraction of inputs in a given cortical area across layers, the threshold 1275 for per structure input signal volume was set at 0.0004. Any structure below this threshold was set 1276 as "0", and no fraction of layer inputs was calculated. This threshold value is higher than $99 \%$ of 1277 input signal volumes measured for structures in the negative dataset, and is equivalent to around 127810 labeled cells based on our comparison of input signal volume and manual counting. We 
reasoned that cortical structures below this threshold have very sparse RV-labeled neurons, which could lead to extreme values when calculating the layer-specific contribution of inputs.

Hierarchical clustering in Figure 2 was conducted using the pvclust package in R. The agglomerative method used in hierarchical clustering was "ward.D", and the distance measure used was correlation. The R software was used for statistical tests and generation of graphs.

\section{Estimation of hierarchical levels}

We first identified a quantitative hierarchical parameter $h$ based on the anatomical features of feedback and feedforward connections, with $h$ calculated as the ratio of layers 2/3/4 input to layers $2 / 3 / 4 / 5$ input. We used the linear regression analysis to estimate a set of hierarchical levels that best predict the measured $h$ values. A model can be specified as $\mathrm{Y}=\mathrm{X} \beta$, wherein $\mathrm{Y}$ is a vector containing the $h$ values of all source areas to each target, $\beta$ contains the estimated hierarchical levels assigned to each area, and $\mathrm{X}$ is the incidence matrix. $\mathrm{X}$ is constructed so that each column corresponds to one of the 43 cortical areas and each row corresponds to a connection between two areas. All of the elements of a row are zero except in the two columns corresponding to the areas participating in the connection, with the source area taking the value of - 1 and the target area taking the value of 1 . The hierarchical level of the primary visual area was set at zero.

1 Reardon, T. R. et al. Rabies Virus CVS-N2c(DeltaG) Strain Enhances Retrograde Synaptic Transfer and Neuronal Viability. Neuron 89, 711-724, doi:10.1016/j.neuron.2016.01.004 (2016).

2 Osakada, F. \& Callaway, E. M. Design and generation of recombinant rabies virus vectors. Nat Protoc 8, 1583-1601, doi:10.1038/nprot.2013.094 (2013).

3 Harris, J. A. et al. Hierarchical organization of cortical and thalamic connectivity. Nature 575, 195-202, doi:10.1038/s41586-019-1716-z (2019).

4 Oh, S. W. et al. A mesoscale connectome of the mouse brain. Nature 508, 207-214, doi:10.1038/nature13186 (2014).

5 Kuan, L. et al. Neuroinformatics of the Allen Mouse Brain Connectivity Atlas. Methods 73, 4-17, doi:10.1016/j.ymeth.2014.12.013 (2015).

6 Wang, Q. et al. The Allen Mouse Brain Common Coordinate Framework: A 3D Reference Atlas. Cell 181, 936-953 e920, doi:10.1016/j.cell.2020.04.007 (2020). 


\section{Supplementary Files}

This is a list of supplementary files associated with this preprint. Click to download.

- SuppleTable1.xlsx

- SuppleTable2.xlsx

- SuppleTable3.xlsx

- SuppleTable4.xlsx

- SuppleTable5.xlsx

- Supplementaryfiguresnumbered.pdf 